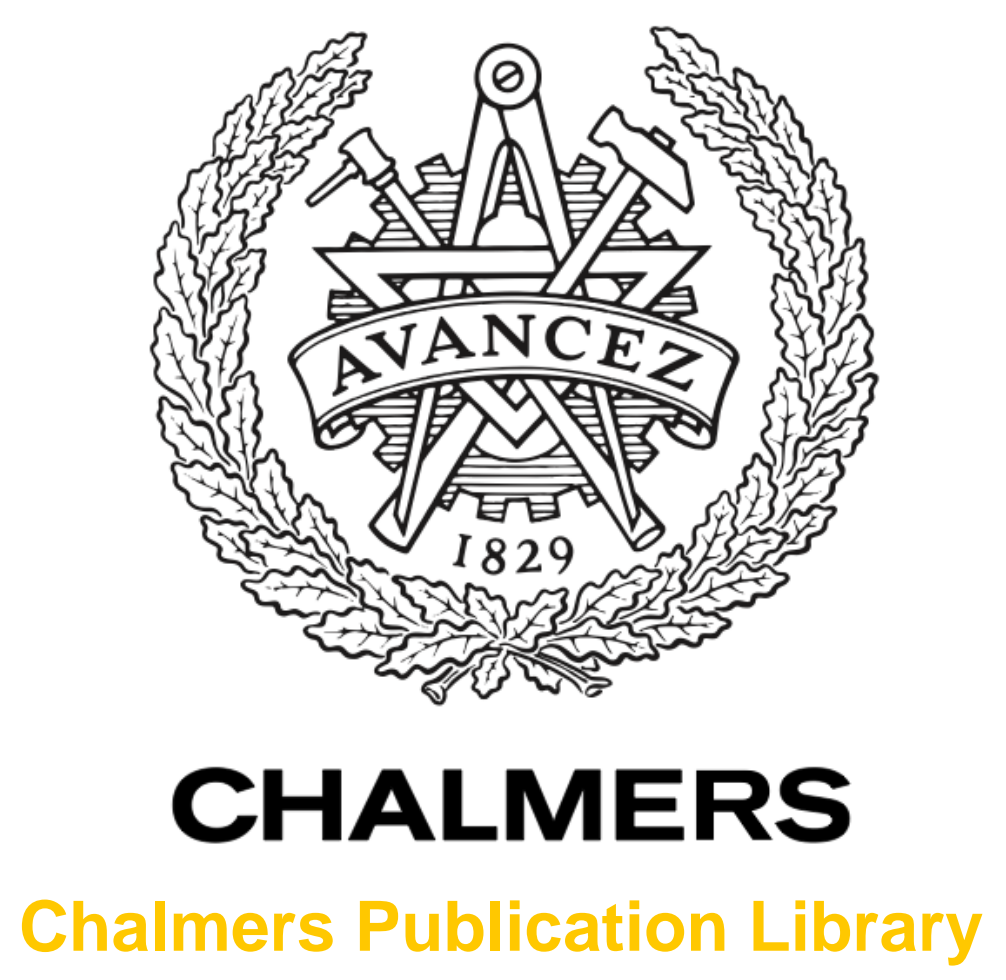

\title{
On the Throughput and Outage Probability of Multi-Relay Networks With Imperfect Power Amplifiers
}

This document has been downloaded from Chalmers Publication Library (CPL). It is the author's version of a work that was accepted for publication in:

leee Transactions on Wireless Communications (ISSN: 1536-1276)

Citation for the published paper:

Makki, B. ; Svensson, T. ; Eriksson, T. et al. (2015) "On the Throughput and Outage

Probability of Multi-Relay Networks With Imperfect Power Amplifiers". Ieee Transactions on Wireless Communications, vol. 14(9), pp. 4994-5008.

http://dx.doi.org/10.1109/twc.2015.2431257

Downloaded from: http://publications.lib.chalmers.se/publication/227719

Notice: Changes introduced as a result of publishing processes such as copy-editing and formatting may not be reflected in this document. For a definitive version of this work, please refer to the published source. Please note that access to the published version might require a subscription. 


\title{
On the Throughput and Outage Probability of Multi-relay Networks with Imperfect Power Amplifiers
}

\author{
Behrooz Makki, Tommy Svensson, Thomas Eriksson and Masoumeh Nasiri-Kenari, Senior Member, IEEE
}

\begin{abstract}
This paper studies the energy-limited performance of multi-relay networks. Taking the properties of the power amplifiers (PAs) into account, we derive closed-form expressions for the optimal power allocation, the outage probability and the throughput in the cases with a sum consumed energy constraint. Moreover, we analyze the diversity and the multiplexing gains of the PA-aware systems. Finally, we investigate the performance of large-scale multi-relay networks and develop efficient multi-relay systems with low cooperation overhead. The numerical and the analytical results show that the inefficiency of the PAs affects the outage probability and the throughput of the multi-relay systems substantially.
\end{abstract}

\section{INTRODUCTION}

The next generation of wireless networks must provide highrate data streams for everyone everywhere at any time. To address the demands, the main strategy persuaded in the last few years is the network densification [1]. One of the promising methods to densify the network is to use many small cheap distributed relay nodes that support the data transmission from the sources to the destinations. This approach is referred to as multi-relaying in the literature [2]-[6].

Multi-relaying is an efficient scheme to increase the service availability and coverage area in dense networks. Compared to the current cellular networks, the relays are expected to cover regions of considerably smaller diameter [3]. Moreover, the relays are responsible for local connections and, depending on the data traffic, might be mostly off. As a result, multi-relaying is an attractive approach, in terms of green communication. However, because the relays consist of simple cheap elements, their hardware impairments, specially the power amplifiers (PAs) efficiency, should be taken into account in the performance analysis [7]-[15]. The problem becomes more important when we remember that in life cycle studies of the state-of-the-art wireless communication systems the radio transmitters have been estimated to be responsible for $70 \%-90 \%$ of the energy consumption during operation; most of the electrical energy is consumed by the final radio frequency (RF) PA stage [16]-[18]. Therefore, designing

Behrooz Makki, Tommy Svensson and Thomas Eriksson are with Chalmers University of Technology, Gothenburg, Sweden, Email: \{behrooz.makki, tommy.svensson, thomase\}@chalmers.se. Masoumeh Nasiri-Kenari is with the Electrical Engineering Department, Sharif University of Technology, Tehran, Iran, Email: mnasiri@sharif.edu.

This work was supported in part by the Swedish Governmental Agency for Innovation Systems (VINNOVA) within the VINN Excellence Center Chase, as well as the VR research link grant on Green Communication via Multirelaying. amplifier-aware data transmission schemes is of fundamental importance [18], [19].

In this paper, we study the data transmission efficiency of the multi-relay systems in the cases with non-ideal (realistic) PAs at the relays. The problem is cast as the optimization of the throughput and the outage probability in the presence of a sum consumed energy constraint on the source and the relays ${ }^{1}$. For symmetric and asymmetric multi-relay setups ${ }^{2}$, with identical and different long-term channel statistics, respectively, we derive closed-form expressions for the optimal power allocation rules and the network outage probability/throughput. Also, we derive necessary conditions for positive diversity and multiplexing gains in a PA-constrained multi-relay network. Finally, we analyze the data transmission efficiency of the large-scale multi-relay networks, with many parallel relay nodes, and propose low-overhead cooperation schemes for multi-relaying. Among the others, some of the interesting conclusions of the paper are as follows:

- The cooperation gain of the multi-relaying systems decreases at low signal-to-noise ratios (SNRs). Therefore, compared to the cooperative multi-relaying, the noncooperative multi-relay setups are preferable at low SNRs because they lead to the same throughput and outage probability with less implementation complexity/signaling overhead.

- With a PA-constrained multi-relay system, a necessary condition to have positive diversity and multiplexing gains is that the number of relays scales with the total consumed power at least logarithmitically.

- A large-scale multi-relay system with no channel state information (CSI) feedback reaches half the ergodic capacity of the non-relay setup with perfect CSI feedback. Therefore, multi-relaying can compensate the lack of CSI in limited feedback schemes.

Moreover, the numerical results show that the inefficiency of the PAs affects the performance of multi-relay networks considerably. For instance, with $M=4$ cooperative relays, the outage probability $10^{-3}$ and common parameter settings

${ }^{1}$ Here, we study the system performance in the cases with sub-codewords of fixed length. Thus, as illustrated in the following, adaptive energy allocation is achieved by updating the sub-codewords powers which are the sub-codewords energies scaled by a constant (length of the sub-codewords).

${ }^{2}$ Symmetric and asymmetric networks are also referred to as homogeneous and heterogeneous networks, respectively, in the literature, e.g., [20], [21]. However, for the sake of clarity, we use the terminologies "symmetric and asymmetric networks" in the following to refer to cases with identical and non-identical long-term statistics of the relay links. 
of the PAs, the imperfection of the PAs results in more than $6 \mathrm{~dB}$ loss in the energy efficiency (see Fig. 3).

It is worth noting that the effect of the PAs on the performance of single- and multi-relay networks have been previously studied in [7]-[15]; designing the amplifieraware receivers for orthogonal frequency-division multiplexing (OFDM) amplify-and-forward (AF) relays, developing multiply-and-forward relays for PA-based two-way cooperative communication and analyzing the outage/error probability of the OFDM AF relays are considered in [7], [8] and [9], [10], respectively, where the results are obtained for the cases with single relays. Moreover, [11]-[15] consider the PA-based multi-relay networks in which the power spectral density [11], the symbol error rate [12], [13], the optimal power allocation [14] and the power efficiency [15] of the network are investigated for different relaying schemes. In these works, the results are obtained for various PA nonlinearity/memory models, for instance, the Bussgang linearization theory, which are different from our PA model. Here, we analyze the system performance for the PA efficiency model given in [22]-[28] which has been validated both analytically and experimentally (the results of [22]-[28] are on non-relay networks with different problem formulations/conclusions from our work). In summary, we consider different PA model, optimization criteria/metrics and problem formulation, compared to [7]-[15], which lead to new analysis/conclusions. Moreover, our discussions on the diversity and multiplexing gains, the optimal power allocation and the large-scale multi-relay networks have not been presented before.

\section{SYSTEM MODEL}

Consider a communication setup with a source, $M$ intermediate relays and a destination. In harmony with, e.g., [10], [12], [29], we ignore the direct link between the source and the destination. The channel coefficient between the source and the $m$-th relay (resp. between the $m$-th relay and the destination) is denoted by $h_{m}$ (resp. $\tilde{h}_{m}$ ). Also, we define $g_{m} \doteq\left|h_{m}\right|^{2}$ and $\tilde{g}_{m} \doteq\left|\tilde{h}_{m}\right|^{2}$ which are referred to as the channel gains in the following. The system performance is studied in blockfading conditions, e.g., [7]-[15], [27]-[31], where the channel coefficients remain constant during the channel coherence time, and then change to other values according to the fading probability density function (PDF). The PDF of a random variable $X$ is represented by $f_{X}$. In each link, the channel coefficient is assumed to be known by the receiver, which is an acceptable assumption in block-fading conditions [7]-[14], [27]-[31]. However, there is no instantaneous CSI available at the transmitters. The codewords are assumed to be constructed by the standard complex Gaussian codes [32, Chapter 9]. Finally, a packet period is defined as the transmission of a message from the source to the destination.

Power amplifier model: We assume an ideal PA for the source, motivated by the fact that the base stations are commonly equipped with considerably stronger PAs than the relays. In the meantime, it is straightforward to extend the results to the cases with non-ideal PA at the source (see Fig. 9 and its corresponding explanations for the cases with a realistic (non-ideal) PA at the source). The relays are equipped with PAs which are modeled as follows.

It has been previously shown that the PA efficiency can be written as [22]

$$
\frac{P}{P^{\mathrm{cons}}}=\epsilon\left(\frac{P}{P^{\mathrm{max}}}\right)^{\vartheta} \Rightarrow P^{\mathrm{cons}}=\alpha P^{1-\vartheta}, \alpha \doteq \frac{\left(P^{\mathrm{max}}\right)^{\vartheta}}{\epsilon} \text {. }
$$

Here, $P, P^{\max }$ and $P^{\text {cons }}$ are the output, the maximum output and the consumed power of the PA, respectively, $\epsilon \in[0,1]$ denotes the maximum power efficiency achieved at $P=P^{\text {max }}$ and $\vartheta$ is a parameter that, depending on the PA classes, varies between $[0,1]$. Moreover, $\alpha \doteq \frac{\left(P^{\max }\right)^{\vartheta}}{\epsilon}$ is an auxiliary variable to simplify the representation of the equations. Thus, using $\alpha=\frac{\left(P^{\max }\right)^{\vartheta}}{\epsilon}$, the following analytical results can be rewritten to represent the system performance based on the PA's maximum output power $P^{\max }$ and efficiency $\epsilon$. Similar models as in (1) have been expressed by [23, eq. (2.14)], [24, eq. (6)], [25, eq. (6) and Table I], [27, eq. (3)] and [28, eq. (3)]. Also, our own efficiency measurements ${ }^{3}$ of different classes of amplifiers have indicated that the equation is indeed quite useful and accurate, e.g., [26]. Therefore, in harmony with [22]-[28], we consider (1) as the PA model. Note that in (1) the parameter $P^{\max }$ has different effects, as it implies a maximum output power constraint $P \leq P^{\max }$ on the relays and also affects the PAs effective efficiency $\epsilon^{\text {eff }}=\epsilon\left(\frac{P}{P^{\max }}\right)^{\vartheta}$. Finally, with an ideal PA we have $\epsilon=1, \vartheta=0, P^{\max } \rightarrow \infty$ in (1).

\section{PROBLEM FORMULATION}

Considering a multi-relay setup with $M$ relays, $Q$ information nats are encoded into a codeword of length $L$ symbols and rate $R=\frac{Q}{L}$. The message is sent from the source to the relays in a frequency slot of bandwidth $w=1$. Let us define $\mathcal{M} \subseteq\{1, \ldots, M\}$ as the set of the relays which decode the source message successfully and $C_{\mathcal{M}}$ as the cardinality of $\mathcal{M}$, i.e., the number of successful relays. Each relay is assigned to a time-frequency chunk of length $L$ and bandwidth $\frac{1}{M}$ (in total, $\frac{L}{M}$ symbols in a chunk). We analyze the system performance for two approaches:

- Centralized cooperative. If relays $\mathcal{M} \subseteq\{1, \ldots, M\}$ decode the codeword correctly, they encode $Q$ nats into a parent codeword of length $\frac{C_{\mathcal{M}} L}{M}$ symbols, divide the parent codeword into $C_{\mathcal{M}}$ sub-codewords of length $\frac{L}{M}$ and each relay uses its own chunk to forward its subcodeword to the destination. The cooperative approach is studied in Section IV.

- Non-centralized non-cooperative. Here, each successful relay encodes the source message into the same codeword of length $\frac{L}{M}$ individually, and forwards the codeword to the destination in a different sub-channel (see Section V for more details).

The motivations for the considered schemes are as follows. As demonstrated in, e.g., [2], two different schemes have been considered for multi-relaying, namely, centralized and noncentralized. In the centralized (cooperative) scheme, a central

\footnotetext{
${ }^{3}$ The measurements have been done in our laboratory at Chalmers [26].
} 
unit coordinates the relays such that each relay knows the message decoding status of the other relays, and the relays transmission rates/powers/coding are adapted jointly [2]. In the non-centralized (non-cooperative) scheme, on the other hand, each relay operates individually forwarding the source message to the destination.

To enjoy the benefits of the cooperative scheme, the channel code should satisfy the following requirements:

1) A parent code that can be punctured into different subcodewords and

2) a decoder decoding the data based on all received signals.

To create such codes, one can follow the same procedure as in Type II (incremental redundancy) automatic repeat request (ARQ) schemes [31], [33], [34] where the parent code is punctured into sub-codewords but, instead of retransmitting the sub-codewords in the successive retransmission rounds of ARQ, they are sent by the relays in different frequency slots. In this way, knowing the message decoding status of the others, each relay can produce the parent codeword individually and pick its own sub-codeword. Note that, for any given rate, the existence of such codes has been previously proved for sufficiently long Gaussian codes, on which we concentrate [33]. Also, there exist several practical code designs, e.g., [35], [36], that satisfy the considered requirements. Moreover, as seen in the following, the non-cooperative scheme can be considered as a special case of the cooperative approach with the same sub-codewords at the data transmitting relays. Thus, as long as the code is sufficiently long, the existence of the code is guaranteed in the non-cooperative scheme as well [33]. Then, as shown in, e.g., [35], [36], the performance of the ARQ-like codes with asymptotically long codes is very close to the ones with finite block-length. Therefore, although the results of the paper are obtained for the cases with long codewords, similar results are expected in the cases with sub-codewords of moderate length. Finally, note that, while Gaussian codes support any transmission rates, in the practical codes the number of relays and the code rate of the noncooperative scheme should be designed such that the relays data rate does not exceed 1.

The destination combines all signals received from the relays to decode the message. In this way, the system throughput is given by

$$
\eta=\frac{Q}{2 L} \operatorname{Pr}(\text { Decoding })=\frac{R}{2}(1-\operatorname{Pr}(\text { Outage })),
$$

where $\operatorname{Pr}($ Outage $)$ is the outage probability, i.e., the probability of the event that the source message is not correctly received by the destination. Also, $\operatorname{Pr}($ Decoding $)=1-$ $\operatorname{Pr}($ Outage $)$ is the successful message decoding probability. For a given rate $R$, the throughput (2) is a monotonic function of the outage probability $\operatorname{Pr}($ Outage). Therefore, we do not need to optimize the throughput and the outage probability separately. Instead, both the maximum throughput and the minimum outage probability are obtained by minimizing the energy-limited outage probability, or equivalently maximizing the energy-limited message decoding probability.
The outage probability is found as

$$
\operatorname{Pr}(\text { Outage })=\sum_{\forall \mathcal{M}} \operatorname{Pr}(\mathcal{M}) \operatorname{Pr}(\text { Outage } \mid \mathcal{M}),
$$

where $\operatorname{Pr}(\mathcal{M})$ is the probability that the source message is correctly decoded by the set of $\mathcal{M} \subseteq\{1, \ldots, M\}$ relays (and not the other relays).

Denoting the source and the $m$-th relay powers by $P$ and $\tilde{P}_{m}$ (in watts), respectively, we can use (1) to find the total consumed energy during a packet transmission period as

$$
\xi=\bar{P} L, \bar{P} \doteq P+\alpha \sum_{\forall \mathcal{M}}\left(\operatorname{Pr}(\mathcal{M}) \sum_{\forall m \in \mathcal{M}} \tilde{P}_{m}^{1-\vartheta}\right),
$$

where $\alpha \tilde{P}_{m}^{1-\vartheta}$ is the consumed power at the $m$-th relay and $\bar{P}$ is defined as the average energy-per-symbol.

Using (2)-(4) for a given rate $R$ and because the throughput is a monotonic function of the outage probability, the energylimited throughput/outage probability optimization problem of the multi-relay network is formed as

$$
\begin{aligned}
& \left.\min _{P, \tilde{P}_{m}, \forall m} \operatorname{Pr} \text { (Outage } \mid P, \tilde{P}_{m}, \forall m\right) \\
& \text { subject to } \quad \bar{P}=\phi, \tilde{P}_{m} \leq P^{\max }, \forall m,
\end{aligned}
$$

where $\phi$ denotes the average energy-per-symbol constraint (in $\mathrm{dB}, 10 \log _{10} \phi$ which, because the noise variance is set to 1 , is referred to as the system SNR as well).

In the following, we study (5). This scenario is of interest in the green communication concept, where the goal is to minimize the total energy required for data transmission, e.g., [37], [38], and also for electricity-bill minimization. For simplicity, we assume the transmission rate $R$ to be previously designed based on, e.g., data structure/coding complexity/quality-ofservice requirements. Therefore, the transmission rate is not considered as an optimization parameter throughout the paper. However, 1) the results are valid for any given transmission rate. Hence, this assumption does not affect the generality of the arguments. Also, 2) it is straightforward to extend the results to the case where, along with the transmission powers, the transmission rate is optimized as well (As an example, see Corollary 2 and Fig. 6 where we derive the optimal rate allocation in the large-scale multi-relay systems).

To study (5), we first need to find the probability terms $\operatorname{Pr}(\mathcal{M})$ and $\operatorname{Pr}($ Outage $\mid \mathcal{M})$ as functions of $P, \tilde{P}_{m}, \forall m$. Having the probabilities, all metrics involved in (5) are represented as functions of the optimization parameters $P, \tilde{P}_{m}, \forall m$. Depending on the number of relays and the PA model, (5) may be a complicated non-convex problem and there may be no closed-form solution for the optimal power terms $P, \widetilde{P}_{m}, \forall m$. Therefore, as stated in Section VI, we may need to use the derived closed-form expressions of the performance metrics and iterative algorithms, to optimize the system performance. An alternative is to implement approximation techniques to derive the optimal power allocation rules, as demonstrated in Properties 2-3.

To close the section, it is interesting to note that with the proposed scheme it might occur that some of the relays subchannels are not utilized for data transmission. However, as 
demonstrated in Subsection IV.B, the amount of the unutilized spectrum resources becomes deterministic in large-scale networks, on which we focus. Therefore, the free spectrum resources can be used for spectrum sharing applications in a cognitive radio manner [1], [39], [40]. Moreover, as seen in the sequel, the spectrum loss is avoided at moderate/high SNRs because most of the relays are always active with high probability. One may consider the scenario where the relays allocated sub-bands are dynamically adapted based on the number of active relays in each slot. We do not consider this scenario for two reasons: 1) Dynamic spectrum and power allocation in distributed large-scale relay networks implies large signaling overhead and implementation complexity at the relays and the destination. 2) With our proposed model, the optimal power allocation rules can be analytically derived, based on the channel long-term statistics. However, the problem of joint dynamic spectrum and power allocation is a non-convex complex problem which does not have a closed-form solution, e.g., [40]. In the meantime, dynamic spectrum and power allocation is an interesting extension of our paper, in which the first challenge would be to fit the block-fading assumption into the variable-bandwidth channels of the active relays. Finally, note that our throughput definition (2) is based on the assumption that the free spectrum of the unsuccessful relays is not utilized by other (cognitive radiobased) systems. The same procedure as in the paper can be applied to analyze the system performance with different definitions of the throughput (see Subsection IV.B for more discussions).

\section{Performance Analysis of the Cooperative Multi-RELAYING APPROACH}

To analyze the system performance, we need to find $\operatorname{Pr}(\mathcal{M})$ and $\operatorname{Pr}($ Outage $\mid \mathcal{M})$. The probability $\operatorname{Pr}(\mathcal{M})$ is given by

$$
\begin{array}{r}
\operatorname{Pr}(\mathcal{M})=\operatorname{Pr}\left(\log \left(1+P g_{m}\right) \geq R, \forall m \in \mathcal{M}\right. \\
\left.\bigcap \log \left(1+P g_{n}\right)<R, \forall n \notin \mathcal{M}\right) .
\end{array}
$$

For Rayleigh fading channels, on which we focus, we have $h_{m} \sim \mathcal{C N}\left(0, \frac{1}{\lambda_{m}}\right)$ and $\tilde{h}_{m} \sim \mathcal{C N}\left(0, \frac{1}{\tilde{\lambda}_{m}}\right), \forall m$. Thus, the PDFs of the channel gains $g_{m}$ and $\tilde{g}_{m}$ are given by $f_{g_{m}}(x)=$ $\lambda_{m} e^{-\lambda_{m} x}, f_{\tilde{g}_{m}}(x)=\tilde{\lambda}_{m} e^{-\tilde{\lambda}_{m} x}$ with $\lambda_{m}$ and $\tilde{\lambda}_{m}, \forall m$, denoting the fading parameters. Hence, for Rayleigh fading channels, (6) is rephrased as

$$
\operatorname{Pr}(\mathcal{M})=e^{-\theta \sum_{\forall m \in \mathcal{M}} \lambda_{m}} \prod_{\forall n \notin \mathcal{M}}\left(1-e^{-\theta \lambda_{n}}\right),
$$

where $\theta \doteq \frac{e^{R}-1}{P}$.

Considering the achievable rates of the parallel Gaussian channels [32, Chapter 9.4] and the fact that different chunks of bandwidth $\frac{1}{M}$ are used by the relays to send the sub-codewords to the destination, the probability $\operatorname{Pr}($ Outage $\mid \mathcal{M})$ is found as

$$
\operatorname{Pr}(\text { Outage } \mid \mathcal{M})=\operatorname{Pr}\left(\frac{1}{M} \sum_{\forall m \in \mathcal{M}} \log \left(1+M \tilde{g}_{m} \tilde{P}_{m}\right)<R\right) \text {, }
$$

in the cooperative approach. Property 1 provides an exact expression and bounds for (8).

Property 1. The probabilities $\operatorname{Pr}($ Outage $\mid \mathcal{M})$ are given by

$$
\begin{aligned}
& \operatorname{Pr}(\text { Outage } \mid \mathcal{M})=1-e^{\frac{1}{M} \sum_{\forall m \in \mathcal{M}} \frac{\tilde{\lambda}_{m}}{\bar{P}_{m}} \times} \\
& \mathcal{H}_{1, C_{\mathcal{M}}+1}^{C_{\mathcal{M}}+1,0}[\left.\frac{e^{M R}}{M^{\mathcal{M}}} \prod_{\forall m \in \mathcal{M}}\left(\frac{\tilde{\lambda}_{m}}{\tilde{P}_{m}}\right)\right|_{(0,1,0)}, \underbrace{\left(1,1, \frac{\tilde{\lambda}_{m}}{M \tilde{P}_{m}}\right), \ldots,}_{\forall m \in \mathcal{M}},
\end{aligned}
$$

where $\mathcal{H}_{s_{1}, s_{2}}^{s_{3}, s_{4}}[. \mid \cdot]$ is the generalized upper incomplete Fox' $\mathrm{H}$ function [41], [42]. Also, the probabilities are bounded by

$$
\begin{aligned}
& \operatorname{Pr}(\text { Outage } \mid \mathcal{M}) \geq U_{\mathcal{M}}, \\
& U_{\mathcal{M}} \doteq \sum_{\forall m \in \mathcal{M}} a_{m}\left(1-e^{-\frac{\tilde{\lambda}_{m} C_{\mathcal{M}}}{M \tilde{P}_{m}}\left(e^{\frac{M R}{C_{\mathcal{M}}}}-1\right)}\right), \\
& a_{m} \doteq \prod_{\forall n \in \mathcal{M}, n \neq m}\left(1-\frac{\tilde{\lambda}_{m} \tilde{P}_{n}}{\tilde{\lambda}_{n} \tilde{P}_{m}}\right)^{-1}, \frac{\tilde{\lambda}_{n}}{\tilde{P}_{n}} \neq \frac{\tilde{\lambda}_{m}}{\tilde{P}_{m}}, n \neq m .
\end{aligned}
$$

Proof. The probability (9) is obtained by defining the random variables $\tilde{\gamma}_{m}=M \tilde{P}_{m} \tilde{g}_{m}, \forall m$, and appropriate parameter setting in the outage expression [41, eq. 18]. Note that the generalized upper incomplete Fox'H function has an efficient MATHEMATICA implementation [41, Appendix B].

Considering the Jensen's inequality, the probability $\operatorname{Pr}($ Outage $\mid \mathcal{M})$ is bounded by

$$
\begin{aligned}
& \operatorname{Pr}(\text { Outage } \mid \mathcal{M}) \stackrel{(a)}{\geq} \operatorname{Pr}\left(\log \left(1+\frac{1}{C_{\mathcal{M}}} \sum_{\forall m \in \mathcal{M}} \tilde{\gamma}_{m}\right) \leq \frac{M R}{C_{\mathcal{M}}}\right) \\
& \stackrel{(b)}{=} \int_{0}^{C_{\mathcal{M}}\left(e^{\frac{M R}{C_{\mathcal{M}}}}-1\right)} \mathcal{L}^{-1}\left\{\frac{1}{\prod_{\forall m \in \mathcal{M}}\left(1+\frac{M \tilde{P}_{m} s}{\tilde{\lambda}_{m}}\right)}\right\} \mathrm{d} z \\
& \stackrel{(c)}{=} \sum_{\forall m \in \mathcal{M}} \int_{0}^{C_{\mathcal{M}}\left(e^{\frac{M R}{C_{\mathcal{M}}}}-1\right)} \frac{\tilde{\lambda}_{m} a_{m}}{M \tilde{P}_{m}} e^{-\frac{\tilde{\lambda}_{m}}{M \tilde{P}_{m}} z} \mathrm{~d} z=U_{\mathcal{M}}
\end{aligned}
$$

Here, $(a)$ is obtained by the Jensen's inequality and the concavity of $\log (1+x)$ in $x$. Then, $(b)$ follows from the fact that the PDF of the sum of independent random variables is obtained by the convolution of their PDFs and $\mathcal{L}\left\{f_{\tilde{\gamma}_{m}}\right\}=\left(1+\frac{M \tilde{P}_{m} s}{\tilde{\lambda}_{m}}\right)^{-1}$ with $\mathcal{L}$ and $\mathcal{L}^{-1}$ being the Laplace transform and its inverse, respectively. Moreover, $(c)$ comes from partial fraction of $S_{\mathcal{M}}(s)=\prod_{\forall m \in \mathcal{M}}\left(1+\frac{M \tilde{P}_{m} s}{\tilde{\lambda}_{m}}\right)^{-1}$ with fraction coefficients $a_{m} \doteq \prod_{\forall n \in \mathcal{M}, n \neq m}\left(1-\frac{\tilde{\lambda}_{m} \tilde{P}_{n}}{\tilde{\lambda}_{n} \tilde{P}_{m}}\right)^{-1}$. Finally, (11) is based on the assumption that $S_{\mathcal{M}}(s)$ has $C_{\mathcal{M}}$ first-order poles, which is the case in asymmetric networks. Straightforward modifications should be applied in the cases with poles of order $>1$ (see (14)).

Using the probabilities $\operatorname{Pr}(\mathcal{M})$ and $\operatorname{Pr}($ Outage $\mid \mathcal{M})$, we can express the throughput, the outage probability and the consumed energy-per-symbol, which are involved in (5), as functions of $P, \tilde{P}_{m}, \forall m$, and analyze the system performance (for simulation results, see Section VI). 


\section{A. Symmetric Networks}

In this subsection, we study the system performance in symmetric channels. Our reasons for selecting the symmetric setups are 1) the analytical results can be derived/followed. Also, 2) as seen in the following, the simulation results of the symmetric channels are not sensitive to parameter settings. This makes it possible to analyze the effect of power amplifiers on the system performance in detail. Finally, 3) the performance analysis of symmetric multi-relay networks is of interest because the derived results can be also utilized to investigate the performance of antenna selection-based multiple-antenna single-relay systems in the presence of nonideal power amplifiers ${ }^{4}$. Moreover, the symmetric networks are useful in the cases where the relays are almost at the same distances from the source and also from the destination.

In symmetric networks, we have $\lambda_{m}=\lambda, \tilde{\lambda}_{m}=\tilde{\lambda}, \forall m$, which rephrases (7) as

$$
\operatorname{Pr}(\mathcal{M})=e^{-C_{\mathcal{M}} \lambda \theta}\left(1-e^{-\theta \lambda}\right)^{M-C_{\mathcal{M}}} .
$$

Also, because in the optimal case the symmetric relays select the same transmission power $\tilde{P}_{m}=\tilde{P}, \forall m$, the average energy-per-symbol and the bound (10) are changed to

$$
\begin{aligned}
\bar{P} & =P+\alpha \tilde{P}^{1-\vartheta} \sum_{\forall \mathcal{M}} \operatorname{Pr}(\mathcal{M}) C_{\mathcal{M}} \\
& =P+\alpha \tilde{P}^{1-\vartheta} \sum_{m=1}^{M} m\left(\begin{array}{l}
M \\
m
\end{array}\right) e^{-m \lambda \theta}\left(1-e^{-\lambda \theta}\right)^{M-m}
\end{aligned}
$$

and

$$
\begin{aligned}
\operatorname{Pr}(\text { Outage } \mid \mathcal{M}) & \geq \int_{0}^{\frac{C_{\mathcal{M}}}{M \tilde{P}}\left(e^{\frac{M R}{C_{\mathcal{M}}}}-1\right)} \mathcal{L}^{-1}\left\{\frac{1}{\left(1+\frac{s}{\tilde{\lambda}}\right)^{C_{\mathcal{M}}}}\right\} \mathrm{d} z \\
& =1-\frac{\Gamma\left(C_{\mathcal{M}}, \frac{C_{\mathcal{M}} \tilde{\lambda}}{M \tilde{P}}\left(e^{\frac{M R}{C_{\mathcal{M}}}}-1\right)\right)}{\Gamma\left(C_{\mathcal{M}}\right)}
\end{aligned}
$$

respectively, where $\Gamma(n, x)=\int_{x}^{\infty} t^{n-1} e^{-t} \mathrm{~d} t$ denotes the incomplete Gamma function and $\left(\begin{array}{l}n \\ k\end{array}\right)$ is the " $n$ choose $k$ " operator.

As mentioned before, the energy-limited outage probability/throughput optimization problem (5) does not have a closed-form solution in general. For this reason, Properties 2 and 3 discuss the high and the low SNR performance of the PA-constrained multi-relay system, respectively. Specially, 1) Property 2 obtains closed-form expressions for the optimal power allocation, throughput and outage probability at high SNRs. Also, 2) the property derives a necessary condition for positive diversity gain in PA-based multi-relay networks. Note that the high-SNR regime is of interest in the PA-based multirelay setups because 1) with high powers most of the relays become active, with high probability, and the relays spectrum loss is avoided. Also, 2) the PA's maximum effective efficiency

\footnotetext{
${ }^{4}$ As opposed to the single-antenna multi-relay setups, the number of active relaying terminals is decided by the antenna selection algorithm in a multiple-antenna single-relay system. This point affects the sum power and the outage probability/throughput expressions. However, the results of Subsection IV.A, with straightforward modifications, can be used to analyze the outage probability/throughput of the amplifier-aware multiple-antenna single-relay systems.
}

$\epsilon^{\text {eff }}=\epsilon\left(\frac{P}{P^{\max }}\right)^{\vartheta}$ is achieved at $P=P^{\max }$ (see (1)), which is the relays power at high SNRs. On the other hand, the low-SNR regime, studied in Property 3 and Subsection IV.B, is interesting in, e.g., large-scale setups when the number of relays increases.

Property 2: Considering the high-SNR regime, the following points are valid for the PA-constrained multi-relay system:

1) Independently of whether the metric of interest is the outage probability or the throughput, the optimal power allocation rule tends towards

$$
\left\{\begin{array}{l}
P=\phi-\frac{M P^{\max }}{\epsilon} \\
\tilde{P}=P^{\max }
\end{array}\right.
$$

2) The high-SNR outage probability and throughput of the multi-relay system are

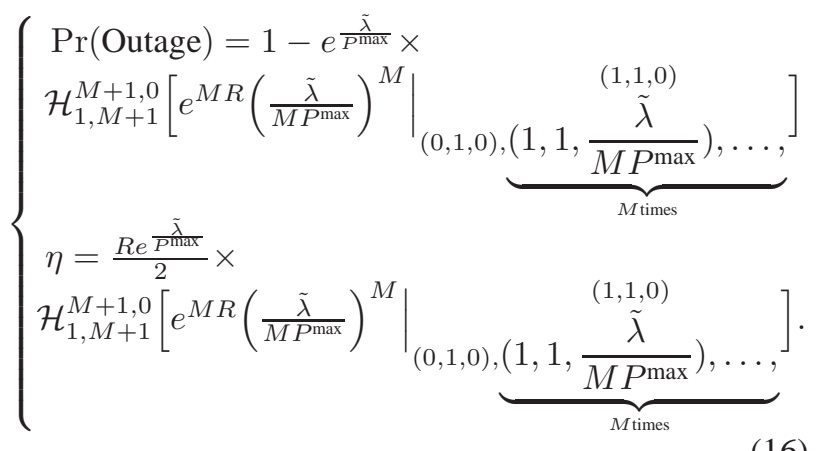

3) For a given number of relays, the diversity gain of the PAconstrained multi-relay setup is zero. A necessary condition to have diversity gain $d>0$ is that the number of relays scales with the total consumed energy-per-symbol by at least $M=\log _{\theta^{\max }}\left(\phi^{-d}\right), \theta^{\max } \doteq \frac{e^{R}-1}{P^{\max }}, \theta^{\max }<1$. That is, to have a positive diversity gain in the PA-based multi-relay system the number of relays should be scaled with the total consumed energy at least logarithmically.

Proof. Considering $\phi \rightarrow \infty$ in (5) and because the throughput and the outage probability are monotonic functions of the transmission power, we have $\tilde{P}=P^{\max }$ and $\operatorname{Pr}(\mathcal{M})=$ $0, \mathcal{M} \neq\{1, \ldots, M\}$, at high SNRs. Hence, from (13) and $\alpha=\frac{\left(P^{\max }\right)^{\vartheta}}{\epsilon}$, the high-SNR optimal power allocation rule of the PA-constrained multi-relay approach tends towards (15) when $\phi \rightarrow \infty$. Note that the same optimal power allocation as in (15) holds for asymmetric networks as well.

Part 2 is a consequence of part 1 where using (2), (3), (9), (15) and $\operatorname{Pr}(\mathcal{M})=0, \mathcal{M} \neq\{1, \ldots, M\}$, at high SNRs, results in (16). As expected, the high-SNR performance of the PA-constrained system is determined by the maximum output power of the PAs. Hence, the outage probability becomes independent of the total energy-per-symbol constraint $\phi$ at high SNRs, and the diversity gain $D=-\lim _{\phi \rightarrow \infty} \frac{\log (\operatorname{Pr}(\text { Outage }))}{\log \phi}$ [43, eq. 14], i.e., the negative of the slope of the outage probability curve at high SNRs, is zero, if the number of relays is finite. 
To have a positive diversity gain $d$, we use (14) to write

$$
\begin{aligned}
D & =-\lim _{\phi \rightarrow \infty} \frac{\log (\operatorname{Pr}(\text { Outage }))}{\log \phi} \\
& \stackrel{(d)}{\leq}-\lim _{\phi \rightarrow \infty} \frac{\log \left(1-\frac{\Gamma\left(M, \tilde{\lambda} \theta^{\max }\right)}{\Gamma(M)}\right)}{\log \phi} \\
& \stackrel{(e)}{=}-\lim _{\phi \rightarrow \infty} \frac{\log \left(1-e^{-\tilde{\lambda} \theta^{\max }} \sum_{j=0}^{M-1} \frac{\left(\tilde{\lambda} \theta^{\max }\right)^{j}}{j !}\right)}{\log \phi} \\
& \stackrel{(f)}{=}-\lim _{\phi \rightarrow \infty} \frac{\log \left(1-e^{-\tilde{\lambda} \theta^{\max }}\left(e^{\tilde{\lambda} \theta^{\max }}-\mathcal{O}\left(\left(\theta^{\max }\right)^{M}\right)\right)\right)}{\log \phi} \\
& =-\lim _{\phi \rightarrow \infty} \frac{\log \left(\left(\theta^{\max }\right)^{M}\right)}{\log \phi} \stackrel{(g)}{=} d .
\end{aligned}
$$

Here, $(d)$ is obtained by defining $\theta^{\max } \doteq \frac{e^{R}-1}{P^{\max }}$ and using (14) with $\mathcal{M}=\{1, \ldots, M\}, \tilde{P}=P^{\max }$, at high SNRs. Then, $(e)$ comes from the definition of incomplete Gamma function $\Gamma(n, x)=\Gamma(n) e^{-x} \sum_{j=0}^{n-1} \frac{x^{j}}{j !}$ with "!" representing the factorial operator. Also, $(f)$ is obtained by the $(M-1)$ th order Taylor expansion of $e^{x}$ with $\mathcal{O}($.$) denoting the order$ operator and $\theta^{\max }<1$. Finally, $(g)$ holds if $M=\log _{\theta^{\max }}\left(\phi^{-d}\right)$, as stated in part 3 of the property. Note that the necessity of the condition follows from the inequality in (17).

Property 3: For a given rate $R$, the low-SNR optimal, in terms of the throughput and the outage probability, power allocation rule of the PA-constrained multi-relay network tends towards

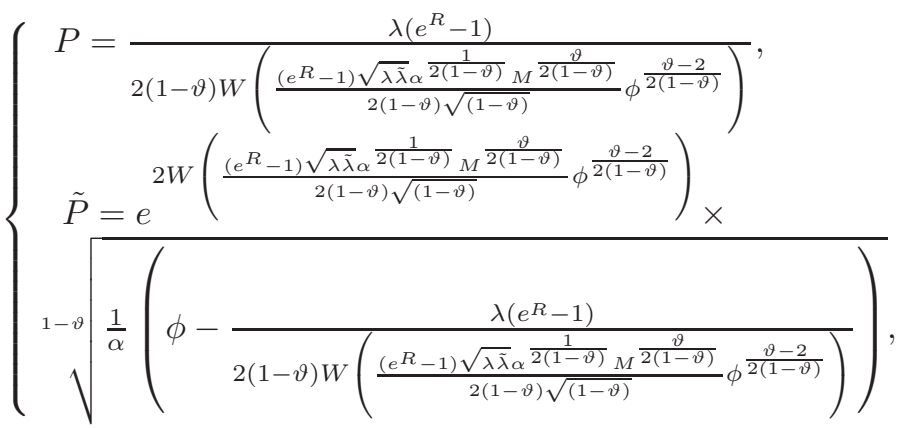

from which the optimal throughput and outage probability are obtained. In (18), $W(x)$ is the Lambert W function [44].

Proof. See Appendix A.

The low-SNR power allocation of asymmetric networks is presented in Appendix B. Also, as another low-SNR approximation, we can use (A.1) in the appendix to write

$$
\tilde{P} \simeq \phi^{\delta} \alpha^{-\delta} M^{-\delta} e^{\frac{\delta \lambda\left(e^{R}-1\right)}{P}}
$$

which, following the same procedure as in (A.3) and (A.4), results in

$$
\left\{\begin{array}{c}
P=\frac{\lambda\left(e^{R}-1\right)}{(1-\vartheta) \log \left(\frac{\tilde{\lambda}\left(e^{R}-1\right) M^{\frac{\vartheta}{1-\vartheta}}}{(1-\vartheta)} 1-\sqrt{\frac{\alpha}{\phi}}\right)} \\
\tilde{P}=\frac{\tilde{\lambda}\left(e^{R}-1\right)}{M(1-\vartheta)} \sqrt[1-\vartheta]{1-\frac{\lambda\left(e^{R}-1\right)}{\phi(1-\vartheta) \log \left(\frac{\tilde{\lambda}\left(e^{R}-1\right) M^{\frac{\vartheta}{1-\vartheta}}}{(1-\vartheta)} \sqrt[1-\vartheta]{\frac{\alpha}{\phi}}\right)}} .
\end{array}\right.
$$

Utilizing the power allocation rules (18) and (20), we can approximate the low-SNR throughput and outage probability of the PA-constrained multi-relay system. Intuitively, the power allocation rule (15) of Property 2 (resp. the approximation scheme of (A.1) in Property 3 and (19)) indicates that at high SNRs (resp. low SNRs) higher powers should be assigned to the source compared to the relays (resp. the relays should receive higher powers compared to the source). For more intuitions on the optimal power allocation, see Fig. 4 and its corresponding explanations. Also, the tightness of the approximations are verified in Fig. 2.

\section{B. Large-Scale Symmetric Networks}

As mentioned before, the main trend in the next generation of wireless networks is to densify the network by, e.g., implementation of many relays. Also, as highlighted in Property 2 part 3, an efficient method to compensate the PAs imperfection is to increase the number of relays. In this part, we investigate the data transmission efficiency of the PA-aware multi-relay network in the cases with high number of relays.

Letting the number of relays $M$ grow asymptotically in a large-scale multi-relay network, the number of relays that correctly decode the source message converges to $M \operatorname{Pr}(\log (1+$ $P g) \geq R)=M e^{-\lambda \theta}$, according to the law of large numbers. Therefore, due to the symmetry in the network, we have

$$
\operatorname{Pr}(\text { Outage })=\operatorname{Pr}\left(\frac{1}{M} \sum_{i=1}^{M e^{-\lambda \theta}} \log \left(1+M \tilde{P} \tilde{g}_{i}\right)<R\right)
$$

Moreover, for large $M$ 's, the outage probability (21) is found as

$$
\begin{aligned}
\operatorname{Pr}(\text { Outage }) & =\operatorname{Pr}\left(\frac{1}{M} \sum_{m=1}^{M e^{-\lambda \theta}} Z_{m} \leq R\right) \\
& \stackrel{(h)}{=} \frac{1}{2}\left(1+\operatorname{erf}\left(\frac{\sqrt{M e^{-\lambda \theta}}\left(R e^{\lambda \theta}-\mu\right)}{\sqrt{2 \sigma^{2}}}\right)\right),
\end{aligned}
$$

where $\operatorname{erf}(x)=\frac{2}{\sqrt{\pi}} \int_{0}^{x} e^{-t^{2}} \mathrm{~d} t$ is the error function. Here, $(h)$ follows from the central limit theorem where the random variable $Z \doteq \frac{1}{M e^{-\lambda \theta}} \sum_{m=1}^{M e^{-\lambda \theta}} Z_{m}$, with $Z_{m} \doteq \log (1+$ $\left.M \tilde{P} \tilde{g}_{m}\right)$, converges in distribution to the Gaussian variable $\mathcal{N}\left(\mu, \frac{\sigma^{2}}{M e^{-\lambda \theta}}\right)$ following the mean and variance

$$
\begin{aligned}
\mu & =E\left\{Z_{m}\right\}=\int_{0}^{\infty} \tilde{\lambda} e^{-\tilde{\lambda} x} \log (1+M \tilde{P} x) \mathrm{d} x \\
& =e^{\frac{\tilde{\lambda}}{M \tilde{P}}} \operatorname{Ei}\left(-\frac{\tilde{\lambda}}{M \tilde{P}}\right)
\end{aligned}
$$




$$
\begin{aligned}
& \sigma^{2}=\rho-\mu^{2}, \\
& \rho=E\left\{Z_{m}^{2}\right\}=\int_{0}^{\infty} \tilde{\lambda} e^{-\tilde{\lambda} x} \log (1+M \tilde{P} x)^{2} \mathrm{~d} x \\
& \stackrel{(i)}{=} 2 M \tilde{P} \int_{0}^{\infty} \frac{e^{-\tilde{\lambda} x}}{1+M \tilde{P} x} \log (1+M \tilde{P} x) \mathrm{d} x \\
& \stackrel{(j)}{\simeq} 2 M^{2} \tilde{P}^{2} \int_{0}^{\beta} \frac{x e^{-\tilde{\lambda} x}}{1+M \tilde{P} x} \mathrm{~d} x \\
& +2 \sum_{n=1}^{\infty} \frac{(-1)^{n+1}(M \tilde{P})^{n}}{n} \int_{\beta}^{\infty} e^{-\tilde{\lambda} x} x^{n-1} \mathrm{~d} x \\
& =2\left(e^{\frac{\tilde{\lambda}}{M \tilde{P}}}\left(\operatorname{Ei}\left(\frac{-\tilde{\lambda}}{M \tilde{P}}\right)-\operatorname{Ei}\left(-\beta \tilde{\lambda}-\frac{\tilde{\lambda}}{M \tilde{P}}\right)\right)+1-e^{-\beta \tilde{\lambda}}\right) \\
& +2 \sum_{n=1}^{\infty} \frac{(-1)^{n+1}}{n}\left(\frac{M \tilde{P}}{\tilde{\lambda}}\right)^{n} \Gamma(n, \beta \tilde{\lambda}), \forall \beta .
\end{aligned}
$$

Here, $E\{$.$\} denotes the expectation operator. Also, (i)$ is obtained by partial integration. Then, $(j)$ consists of two integration parts where the first one comes from $\log (1+x)=x$ for small $x$ 's, and the second one is obtained by the Taylor expansion of the logarithmic term and the approximation $\frac{1}{1+M \tilde{P} x} \simeq \frac{1}{M \tilde{P} x}$ for high values of $M \tilde{P}$ and $x \geq \beta$. Note that the approximation is very tight for every small value of $\beta$. Finally, the last equality in (24) follows from the definition of the exponential integration function $\operatorname{Ei}(x)=\int_{x}^{\infty} \frac{e^{-t} \mathrm{~d} t}{t}$ and the incomplete Gamma function.

Considering (22)-(24) for a given rate $R$ and because $M e^{-\lambda \theta}$ relays are always active in a large-scale setup, the optimization problem (5) is rephrased as

$$
\begin{aligned}
& \min _{P, \tilde{P}} \frac{e^{-\frac{\lambda\left(e^{R}-1\right)}{2 P}}\left(R e^{\lambda \frac{e^{R}-1}{P}}-\mu\right)}{\sigma} \\
& \text { subject to } P+M \alpha e^{-\frac{\lambda}{P}\left(e^{R}-1\right)} \tilde{P}^{1-\vartheta}=\phi, \tilde{P} \leq P^{\max },
\end{aligned}
$$

which, using $\mu$ and $\sigma$ in (23) and (24), can be solved numerically or analytically. The optimization problem (25) is based on (22) and the fact that $\operatorname{erf}(x)$ is an increasing function of $x$. In the following, we study (25) in the high- and low-SNR regimes.

Corollary 1: High-SNR outage probability and throughput of a large-scale multi-relay system converges to

$$
\begin{aligned}
& \operatorname{Pr}(\text { Outage })=\frac{1}{2}\left(1+\operatorname{erf}\left(\frac{\sqrt{M}\left(R-\mu^{\max }\right)}{\sqrt{2} \sigma^{\max }}\right)\right), \\
& \eta=\frac{R}{4}\left(1-\operatorname{erf}\left(\frac{\sqrt{M}\left(R-\mu^{\max }\right)}{\sqrt{2} \sigma^{\max }}\right)\right),
\end{aligned}
$$

respectively, where $\mu^{\max }$ and $\sigma^{\max }$ are obtained by setting $\tilde{P}=$ $P^{\max }$ in (23) and (24), respectively.

Proof. Letting $P \rightarrow \infty$ and $\tilde{P}=P^{\max }$ at high SNRs, (2) and (22) are used to rewrite the throughput and the outage probability of the large-scale multi-relay setup as in (26).

Throughout the paper, we studied the system performance for given rates $R$ selected by the network designer. Meanwhile, as mentioned before, we can optimize the transmission rate, in terms of throughput, as well. Corollary 2 studies the throughput in the cases with optimal transmission rates. Particularly, the corollary introduces the multi-relaying as an alternative for limited feedback systems.

Corollary 2: Consider a large-scale multi-relay network with no CSI feedback. At high SNRs, the network reaches half the ergodic capacity of a point-to-point communication setup with perfect CSI at the transmitter and the same output power as the relays sum output power.

Proof. Letting $M \rightarrow \infty$ in (26.ii), the maximum throughput is achieved by $R=\mu^{\max }-\omega, \omega \rightarrow 0$. Then, from (23) and (26.ii), we have $\mu^{\max }=e^{\frac{\tilde{\lambda}}{M P^{\max }}} \operatorname{Ei}\left(-\frac{\tilde{\lambda}}{M P^{\max }}\right)$ which leads to

$$
\eta^{\text {large-scale multi-relay }}=\frac{1}{2} e^{\frac{\tilde{\lambda}}{M P^{\max }}} \operatorname{Ei}\left(-\frac{\tilde{\lambda}}{M P^{\max }}\right),
$$

at high SNRs. Equation (27) is half the ergodic capacity of a Rayleigh fading point-to-point (non-relay) communication setup with perfect CSI at the transmitter and transmission power $M P^{\max }$ which is given by [45, eq. (9)], [46, eq. (47)]

$$
\begin{aligned}
C^{\text {point-to-point }} & =\int_{0}^{\infty} \tilde{\lambda} e^{-\tilde{\lambda} x} \log \left(1+M P^{\max } x\right) \mathrm{d} x \\
& =e^{\frac{\tilde{\lambda}}{M P^{\max }}} \operatorname{Ei}\left(-\frac{\tilde{\lambda}}{M P^{\max }}\right) .
\end{aligned}
$$

Note that 1) the term $\frac{1}{2}$ in (27) is because of the half-duplex relaying. 2) In contrast to (24), there is no approximation in Corollary 2, because the arguments hold for every value of $\sigma^{2}$ in (22). Finally, 3) with the considered data rate and $M \rightarrow \infty$ the outage probability (26.i) goes to zero, the same as in the perfect-CSI point-to-point setup. Thus, as stated in the corollary, multi-relaying can be considered as an efficient scheme to compensate the lack of CSI in limited-feedback schemes, e.g., [45]-[47]. However, compared to the non-relay setup, the multi-relaying implies implementation complexity, overhead and maintenance costs which should be considered in practical systems.

In Property 2, we showed that a necessary condition for a non-zero diversity gain is to let the number of relays grow with the total consumed energy-per-symbol logarithmically. To emphasize the necessity of the large-scale multi-relay systems, Property 4 derives a necessary condition for a positive multiplexing gain which is defined as $r=\lim _{\phi \rightarrow \infty} \frac{\eta}{\log \phi}$ [43, eq. 14], i.e., the slope of the throughput curve at high SNRs.

Property 4: To have a positive multiplexing gain in a nonideal PA-based multi-relay setup, a necessary condition is that the number of relays grows with the total consumed energyper-symbol at least logarithmically.

\section{Proof. See Appendix C.}

The same arguments as in Property 3 can be applied to derive the optimal power allocation rule of the large-scale setups at low SNRs. Using $\log (1+x)=x$ for small $x$ 's, the mean and variance (23) and (24) are rephrased as $\mu=\frac{M \tilde{P}}{\tilde{\lambda}}$ and $\sigma^{2}=\frac{M^{2} \tilde{P}^{2}}{\tilde{\lambda}^{2}}$ at low SNRs. Consequently, following the same 
procedure as in (25), the low-SNR optimal power allocation rule is found as

$$
\begin{aligned}
& \left\{\begin{array}{l}
\min _{P, \tilde{P}} \frac{e^{\frac{-\lambda\left(e^{R}-1\right)}{2 P}}\left(R e^{\frac{\lambda\left(e^{R}-1\right)}{P}}-\frac{M \tilde{P}}{\hat{\lambda}}\right)}{\sqrt{2} \frac{M \tilde{P}}{\lambda}} \\
\text { subject to } P+M \alpha e^{-\frac{\lambda}{P}\left(e^{R}-1\right)} \tilde{P}^{1-\vartheta}=\phi,
\end{array}\right. \\
& \equiv\left\{\begin{array}{l}
\min _{P, \tilde{P}} \frac{\tilde{\lambda} R}{M \tilde{P}} e^{\frac{\lambda\left(e^{R}-1\right)}{2 P}}-e^{-\frac{\lambda\left(e^{R}-1\right)}{2 P}} \\
\text { subject to } \tilde{P}=\frac{(\phi-P)^{\delta}}{M^{\delta} \alpha^{\delta}} e^{\frac{\lambda \delta}{P}\left(e^{R}-1\right)},
\end{array} \Rightarrow\right. \\
& \min _{0 \leq P \leq \phi}\left\{\tilde{\lambda} R \alpha^{\delta} M^{\delta-1}(\phi-P)^{-\delta} e^{\frac{\lambda\left(e^{R}-1\right)}{2 P}(1-2 \delta)}-e^{-\frac{\lambda\left(e^{R}-1\right)}{2 P}}\right\} \\
& \Rightarrow\left\{\begin{array}{l}
P=\frac{\lambda\left(e^{R}-1\right) \vartheta}{(1-\vartheta) \log \left(\tilde{\lambda} R \alpha^{\frac{1}{1-\vartheta}} M^{\frac{\vartheta}{1-\vartheta}}\left(\frac{\vartheta+1}{1-\vartheta}\right)(\phi)^{\frac{1}{\vartheta-1}}\right)} \\
\tilde{P}=\frac{(\phi-P)^{\frac{1}{1-\vartheta}}}{M^{\frac{1}{1-\vartheta}} \alpha^{\frac{1}{1-\vartheta}}} e^{\frac{\lambda}{P(1-\vartheta)}\left(e^{R}-1\right)} .
\end{array}\right.
\end{aligned}
$$

Here, we have used (22) and the fact that the $\operatorname{erf}(x)$ is an increasing function of $x$. Also, due to the low-SNR assumption, we have relaxed the relays maximum power constraint. The last part of (29) comes from the low SNR approximation $(\phi-P)^{-\delta} \simeq \phi^{-\delta}$ and then setting the derivative of the objective function with respect to $P$ equal to zero. Note that, setting the derivatives of $\mathcal{Y}(P)=\tilde{\lambda} R \alpha^{\delta} M^{\delta-1}(\phi-$ $P)^{-\delta} e^{\frac{\lambda\left(e^{R}-1\right)}{2 P}(1-2 \delta)}-e^{-\frac{\lambda\left(e^{R}-1\right)}{2 P}}$ with respect to $P$ equal to zero and using the properties of the PA model (1), it can be easily shown that the problem $\min _{0 \leq P \leq \phi}\{\mathcal{Y}(P)\}$ has a unique solution deriving the globally optimal, in terms of throughput/outage probability, power allocation rule. Having (29), we can find the low-SNR outage probability and throughput as in (22) and (2), respectively.

Finally, it is interesting to note that in a large-scale setup the parent codeword is of fixed-length $e^{-\lambda \theta} L$ which simplifies the coding process significantly, compared to the variable-length coding in the cases with limited number of relays. Moreover, in a large-scale multi-relay network $M\left(1-e^{-\lambda \theta}\right)$ sub-channels remain unused (with probability 1) in each packet period. Therefore, as the amount of the free spectrum is deterministic, it may be exploited for cognitive-radio applications [39], [48]. In the cases with a joint cognitive radio application, one may rewrite the throughput function of the multi-relay network as

$$
\eta=\frac{Q(1-\operatorname{Pr}(\text { Outage }))}{L+L \bar{w}}=\frac{R(1-\operatorname{Pr}(\text { Outage }))}{1+e^{-\lambda \theta}},
$$

where $\bar{w}$ is the expected bandwidth use of the relays and the last equality holds for the large-scale multi-relay setups. However, it should be noted that, while the amount of the free spectrum is deterministic in large-scale setups, the cognitive radio still needs to find the free spectrum gaps via, e.g., spectrum sensing approaches. For this reason, and also to keep the discussions of the paper in harmony, we do not consider (30) in our analysis.

\section{Performance Analysis in the Non-CoOperative Multi-Relaying APPROACH}

In Section IV, we analyzed the system performance in the cooperative multi-relay setup where each relay with successful message decoding transmits part of a parent codeword to the destination. Although this scheme leads to considerable throughput and outage probability improvements, it implies signaling overhead and complexity because each relay needs to know the other relays message decoding status. Also, as the number of successful relays is a random variable in the cases with limited number of relays, the cooperative scheme implies variable-length coding in order to make the parent codeword. In the following, we restudy the system performance in the non-cooperative setup with low signaling overhead/coding complexity.

Consider the case where each successful relay re-encodes the source message into a codeword of length $\frac{L}{M}$, based on a predefined protocol. Therefore, the same codeword is forwarded from the successful relays towards the destination in different sub-channels. The destination performs maximum ratio combining of all received copies of the relays' codeword, which is the optimal decoder in the repetition code-based schemes [49]. In this way, the relays need not to know the message decoding status of the others and they work noncooperatively, which reduces the cooperation overhead remarkably. Moreover, as each relay re-encodes the source message non-cooperatively, the variable-length coding is avoided, simplifying the coding process.

From the analytical perspective, the only difference between the cooperative and non-cooperative schemes is that the conditional outage probability term $\operatorname{Pr}($ Outage $\mid \mathcal{M})$ in (8) is replaced by

$$
\begin{aligned}
& \operatorname{Pr}(\text { Outage } \mid \mathcal{M})^{\text {non-cooperative }} \\
& =\operatorname{Pr}\left(\frac{1}{M} \log \left(1+M \sum_{\forall m \in \mathcal{M}} \tilde{g}_{m} \tilde{P}_{m}\right)<R\right),
\end{aligned}
$$

in the non-cooperative approach. The other analysis such as (2), (3), (4) and (7) remain the same as before. Changing the inequality into equality and implementing appropriate modifications, the same procedure as in (11) is applied to find (31) in asymmetric networks as

$$
\begin{aligned}
& \operatorname{Pr}(\text { Outage } \mid \mathcal{M})^{\text {non-cooperative }}= \\
& \sum_{\forall m \in \mathcal{M}}\left(\prod_{\forall n \in \mathcal{M}, n \neq m}\left(1-\frac{\tilde{\lambda}_{m} \tilde{P}_{n}}{\tilde{\lambda}_{n} \tilde{P}_{m}}\right)^{-1}\right)\left(1-e^{-\frac{\tilde{\lambda}_{m}}{M \tilde{P}_{m}}\left(e^{M R}-1\right)}\right)
\end{aligned}
$$

Also, with a symmetric setup, $\tilde{\lambda}_{m}=\tilde{\lambda}, \tilde{P}_{m}=\tilde{P}, \forall m,(31)$ is rephrased as

$$
\begin{aligned}
& \operatorname{Pr}(\text { Outage } \mid \mathcal{M})^{\text {non-cooperative }}=\operatorname{Pr}\left(\sum_{\forall m \in \mathcal{M}} \tilde{g}_{m} \leq \frac{e^{M R}-1}{M \tilde{P}}\right) \\
& =\int_{0}^{\frac{e^{M R}-1}{M \tilde{P}}} \mathcal{L}^{-1}\left\{\left(1+\frac{s}{\tilde{\lambda}}\right)^{-C_{\mathcal{M}}}\right\} \mathrm{d} z \\
& =1-\frac{\Gamma\left(C_{\mathcal{M}}, \frac{\tilde{\lambda}\left(e^{M R}-1\right)}{M \tilde{P}}\right)}{\Gamma\left(C_{\mathcal{M}}\right)} .
\end{aligned}
$$

Indeed, the cooperative scheme outperforms the noncooperative approach in terms of throughput and outage probability. However, Property 5 compares their data transmission efficiency at low SNRs. Interestingly, the property indicates 
that the cooperation gain decreases at low SNRs. Therefore, compared to the cooperative approach, the non-cooperative model is preferable at low SNRs because it results in the same throughput and outage probability, with less implementation complexity/signaling overhead.

Property 5: The proposed cooperative and non-cooperative multi-relaying approaches lead to the same energy-limited throughput and outage probability at low SNRs.

Proof. See Appendix D.

Figure 8 in Section VI compares the performance of the cooperative and non-cooperative schemes numerically.

\section{SIMULATION RESULTS}

In Figs. 1-9, the simulation results are given for a symmetric network with $\lambda_{m}=\tilde{\lambda}_{m}=1, \forall m$. Then, Fig. 10 shows the system performance in asymmetric systems. Also, we set $R=0.5$ nats-per-channel-use (npcu) in all figures, except in Fig. 6 which presents the system throughput for different transmission rates. The results are presented for different ranges of SNR, defined as $10 \log _{10} \phi$ (see (5)). In harmony with, e.g., [27], [28], we set $\vartheta=0.5$ and $P^{\max }=18 \mathrm{~dB}$, unless otherwise stated. In the meantime, we have checked the results for other parameter settings which show the same qualitative conclusions as the ones presented below. In all figures except Fig. 8, we consider the cooperative multirelaying scheme. Comparison between the cooperative and non-cooperative approaches is given in Fig. 8.

Throughout the paper, we presented different approximation/bounding techniques. The verification of these results is demonstrated in Figs. 1-2, 5-6 and, as seen in the sequel, the analytical results follow the simulations with high accuracy. Then, to avoid too much information in each figure, Figs. 34, 7-10 report only the simulation results. Note that in all simulations we have double-checked the results with the ones obtained analytically.

To optimize the transmission powers, we have implemented different schemes. Using the closed-form expressions of the probabilities, the results are first obtained by exhaustive search on the power terms. Then, for faster convergence, we have repeated the simulations by using the iterative algorithm of [47], and by using "fminsearch" and "fmincon" functions of MATLAB, whenever they are applicable. The results have been obtained for different initial settings and we have tested the fmincon function for "interior-point," "active-set" and "trust-region-reflective" options of the optimization algorithm. In all cases, the results are the same with high accuracy, which is an indication of a reliable result.

The simulation results are presented in different parts as follows.

Verification of Properties 1-3: In Figs. 1-2, we evaluate the accuracy of the results given in Properties 1-3 and the approximation technique (20). The results are obtained for $M=2,3$ relays and $\epsilon=0.65$. As seen in Fig. 1, the lower bound of Property 1 is very tight for a large range of SNRs. Moreover, the same outage probability is achieved by the multi-relay scheme if the probabilities are calculated via Property 1 equation (9) or Monte Carlo simulations, which

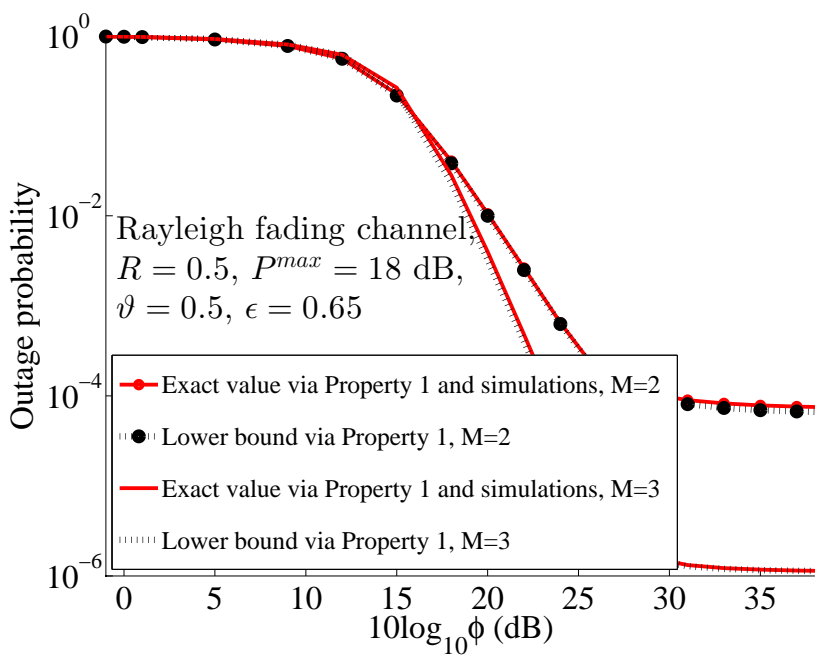

Figure 1. Outage probability vs $10 \log _{10} \phi(\mathrm{dB})$ defined in (5). Rayleigh fading channel, $R=0.5, P^{\max }=18 \mathrm{~dB}, \vartheta=0.5, \epsilon=0.65$.

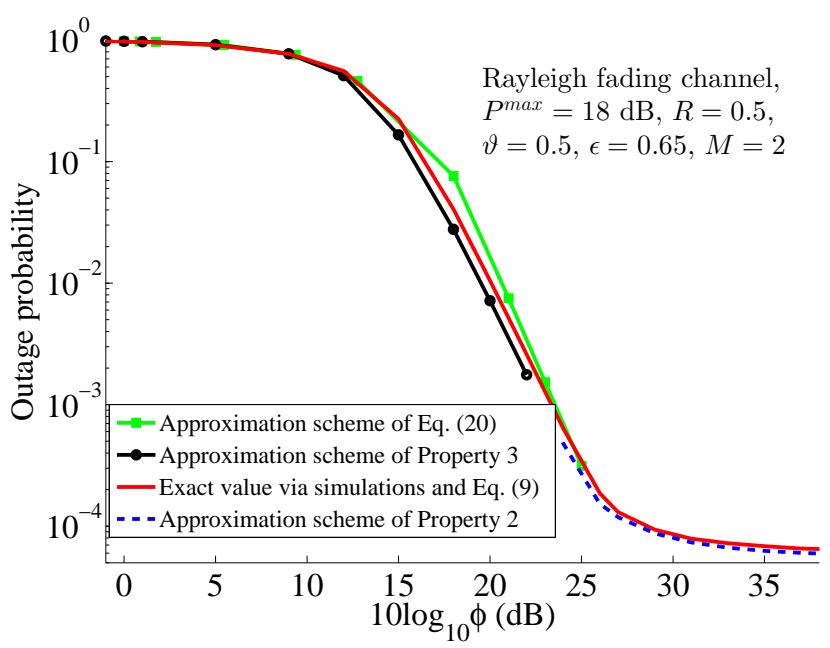

Figure 2. Verification of Properties 2-3 and approximation (20). Rayleigh fading channel, $R=0.5, P^{\max }=18 \mathrm{~dB}, \vartheta=0.5, \epsilon=0.65, M=2$.

is expected because there is no approximation in (9) (Also, see [41]). Furthermore, as shown in Fig. 2, the high-SNR approximation of Property 2 and the low SNR approximations of Property 3/equation (20) follow the exact values obtained via simulations for a large range of SNRs.

Outage probability with ideal and non-ideal PAs: Figure 3 shows the outage probability of the PA-constrained multi-relay setup and compares the results with case where the PAs are assumed to be ideal. For the non-ideal PA, we set $P^{\max }=18$ $\mathrm{dB}, \vartheta=0.5, \epsilon=0.55$, while the ideal PA corresponds to $P^{\max } \rightarrow \infty, \vartheta=0, \epsilon=1$ (see (1)). In harmony with Property 1 part 3 , the outage probability of the multi-relay setup becomes constant at high SNRs when the properties of the PAs are taken into account. That is, the diversity gain (the negative of the slope of the outage probability curve at high SNRs) of the PA-constrained multi-relay setup is zero for finite number of relays. Also, the inefficiency of the PAs leads to remarkable performance degradation at low/moderate SNRs, and the effect of the PAs inefficiency increases with the number of relays $M$. As an example, consider the case with 


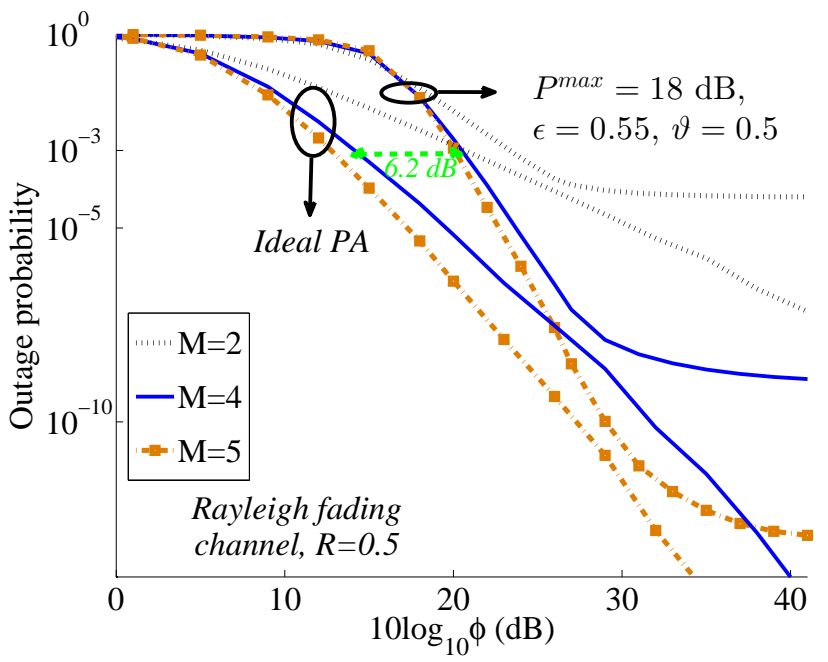

Figure 3. Comparison between the ideal and non-ideal PAs, Rayleigh fading channel, $R=0.5$. For the cases with non-ideal PA, we set $P^{\max }=18 \mathrm{~dB}$, $\vartheta=0.5, \epsilon=0.55$

an outage probability $10^{-3}$ and $M=4$ relays. Then, with the parameter setting of the figure, the imperfection of the PAs results in $6.2 \mathrm{~dB}$ loss in the energy efficiency.

On the optimal power terms: Shown in Fig. 4 are the optimal powers $P$ and $\tilde{P}$, in terms of the outage probability and the throughput. The results are in harmony with the intuitions derived from Properties 2-3; at low SNRs, either none or one of the relays may become active, with high probability. Therefore, high powers are assigned to the active relay. Moreover, as the number of relays increases, the chance of having one successful relay increases. Hence, at low SNRs, a smaller proportion of the power is allocated to the source when the number of relays increases, and the relays' power increases with $M$. Increasing the $\mathrm{SNR}$, more relays may become active and share the energy resources. As a result, depending on the SNR budget and the number of relays, the power allocated to each relay may decrease. Finally, at high SNRs the relays powers are limited by the PAs maximum output power and the source power follows (15) given in Property 2. Also, the source power increases with the SNR budget monotonically, as expected.

On large-scale multi-relaying: Figures 5-6 study the analytical results of the large-scale setups, i.e., when the number of relays increases. As demonstrated in Fig. 5, the outage probability expression (22) and the optimal power allocation rule of (29) are very tight even for limited number of relays. Figure 6a compares the throughput achieved via Monte Carlo simulations and the one obtained by (26.ii) in Corollary 1 for different numbers of relays. Also, shown in Fig. $6 \mathrm{~b}$ is the relative throughput $\Delta=\frac{C^{\text {point-to-point }}}{2 \max _{R}\{\eta\}}$, to validate Corollary 2 . Here, $\max _{R}\{\eta\}$ is the maximum throughput that is achieved by sweeping on the codeword rates $R$, for a given $\mathrm{SNR} /$ maximum output power of the PAs. Also, the results of Fig. 6 are based on sufficiently high SNR assumption so that all relays are active (with probability 1) forwarding messages with power $P^{\max }$. As illustrated in the figures, for small codeword rates, the throughput increases with codeword rate (almost) linearly

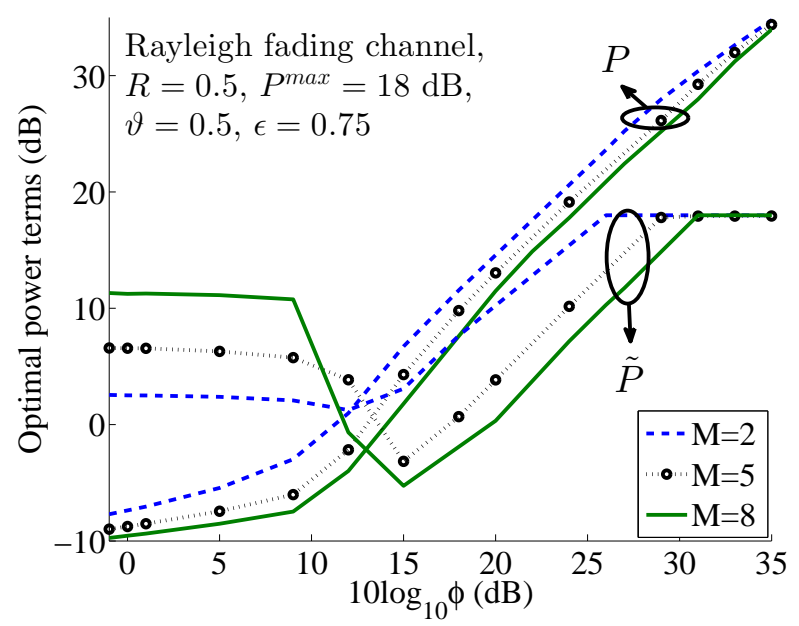

Figure 4. Optimal power terms $P$ and $\tilde{P}$ vs the SNR $10 \log _{10} \phi(\mathrm{dB})$. Rayleigh fading channel, $R=0.5, P^{\max }=18 \mathrm{~dB}, \vartheta=0.5, \epsilon=0.75, M=$ $2,5,8$.

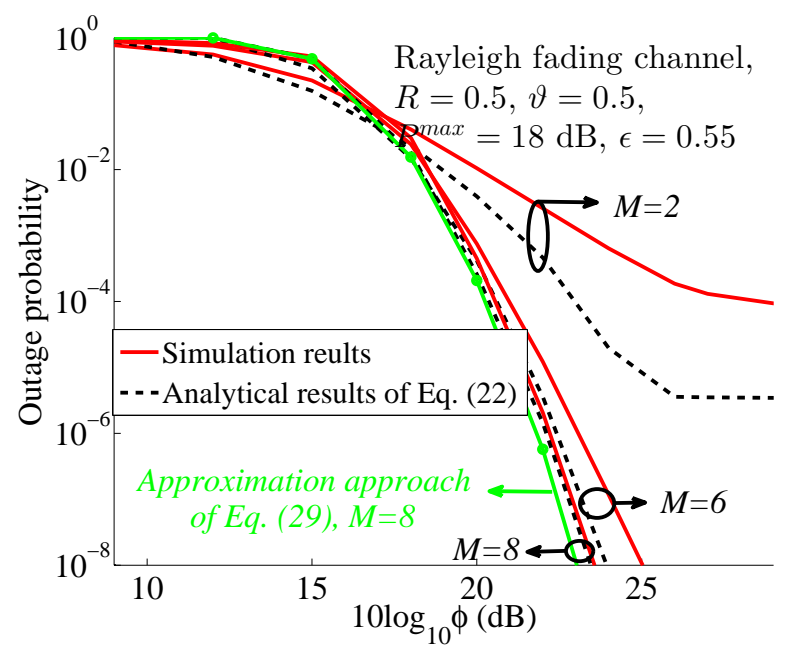

Figure 5. Verification of the analytical results on large-scale multi-relay systems. Rayleigh fading channel, $R=0.5, P^{\max }=18 \mathrm{~dB}, \vartheta=0.5, \epsilon=0.55$.

(Fig. 6a) and the maximum value of the throughput converges to half of the ergodic capacity of the point-to-point nonrelay system as the number of relays increases (Fig. 6b and Corollary 2). Furthermore, at high SNRs, the maximum throughput of the multi-relay setup increases with the number of relays. However, with finite number of relays the peak throughput is limited, i.e., the multiplexing gain is zero, as proved in Property 4. Finally, the approximation technique of Corollary 1 accurately mimics the simulation results and the tightness of the approximation increases with the number of relays $M$ (Fig. 6a).

On the optimal number of relays: As mentioned before, with the proposed scheme it might occur that some of the relays sub-bands are not utilized for data transmission. Therefore, there is a tradeoff between increasing the diversity/the relays message decoding probability and the relays spectrum underutilization. Thus, depending on the SNR budget and the PAs properties, there might be an optimal number of relays minimizing the outage probability. This point is illustrated in 

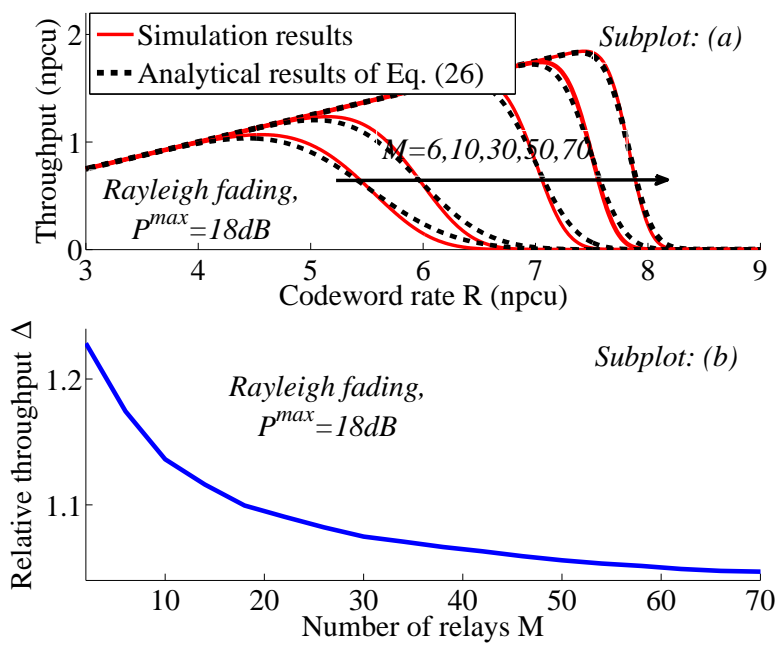

Figure 6. Comparison between the simulation results and the analytical results of (a): Corollary 1 and (b): Corollary 2, Rayleigh fading channel, $P^{\max }=18$ $\mathrm{dB}$. The total SNR is supposed to be large enough so that all relays are active forwarding messages at power $P^{\max }$.

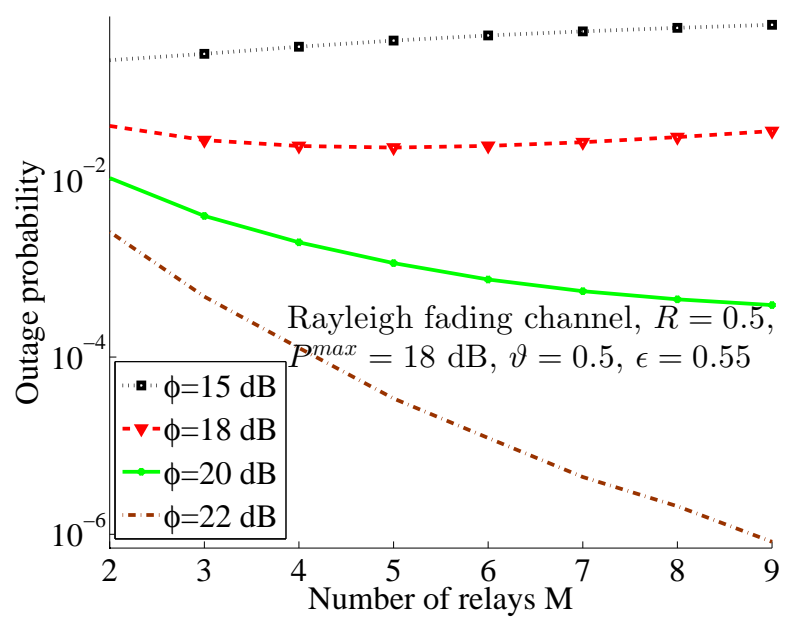

Figure 7. The outage probability of the multi-relay setup for different numbers of relays. Rayleigh fading channel, $R=0.5, P^{\max }=18 \mathrm{~dB}, \vartheta=0.5, \epsilon=$ 0.55 .

Fig. 7 where, as an example, with the parameter settings of the figure the minimum outage probability is obtained by $M=5$ relays at $\phi=18 \mathrm{~dB}$. However, at high SNRs, where with high probability all relays decode the source message correctly and the spectrum under-utilization is avoided, the system performance is improved by increasing the number of relays, i.e., the outage probability decreases with $M$ monotonically (see Fig. 7 for $\phi=20,22 \mathrm{~dB}$ ).

Comparison between the cooperative and non-cooperative multi-relaying schemes: Setting $M=2$ and 4, Fig. 8 compares the outage probability achieved by the cooperative and non-cooperative multi-relaying schemes. Here, we set $R=0.5, \epsilon=0.55, P^{\max }=18 \mathrm{~dB}$. As seen, the difference between the performance of the cooperative and the noncooperative schemes is negligible at low SNRs/few number of relays, in harmony with Property 5. However, the cooperative scheme outperforms the non-cooperative model, in terms of

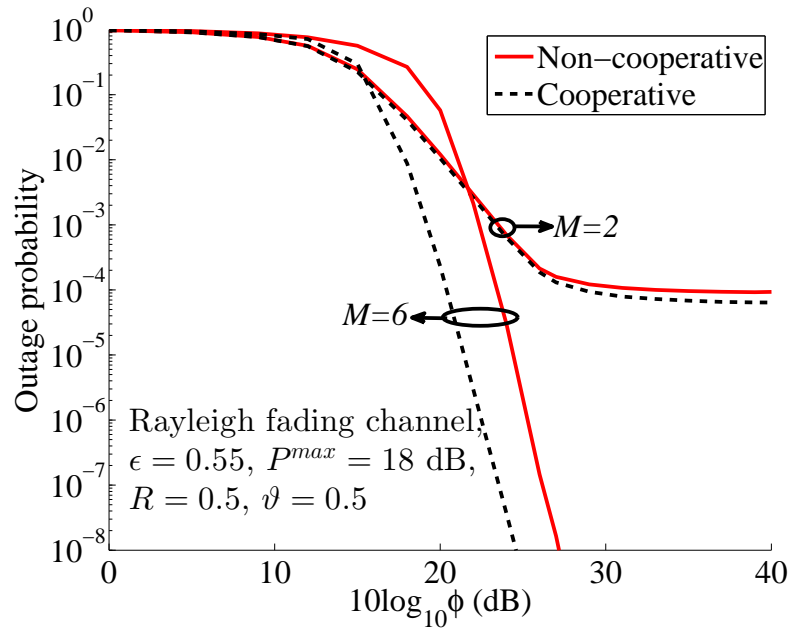

Figure 8. Comparison between the cooperative and non-cooperative multirelaying schemes. Rayleigh fading channel, $R=0.5, P^{\max }=18 \mathrm{~dB}, \vartheta=$ $0.5, \epsilon=0.55$.

the outage probability/throughput, and the difference between the two schemes increases with the SNR/number of relays.

On the effect of the source PA: Throughout the paper, we assumed an ideal PA for the source, motivated by the fact that the base stations commonly utilize considerably stronger and more expensive PAs compared to the relays. However, it is straight forward to extend the results of the paper to the cases with non-ideal PAs at the source. As an example, Fig. 9 shows the relative throughput which is defined as $\hat{\Delta}=\frac{\hat{\eta}}{\eta}$ with $\hat{\eta}$ being the throughput achieved in the case with a nonideal PA at source having parameters $\hat{P}^{\text {max }}, \hat{\vartheta}, \hat{\epsilon}$ that affect (5) correspondingly (In the cases with different PAs of the source, the parameters of the relays' PAs are set to $P^{\max }=$ $18 \mathrm{~dB}, \vartheta=0.5, \epsilon=0.65)$. As illustrated, the inefficiency of the source PA affects the relative throughput considerably at low SNRs. However, the relative throughput degradation, due to the imperfection of the source PA, is negligible as the SNR increases.

Performance analysis in asymmetric networks: While (2)(11), Properties 1-2, 4 and Appendix B derive the results for asymmetric networks, we have mainly concentrated on the symmetric setups in the simulations. Property 1 and the same procedure as in, e.g., [47, Algorithm 1] can be utilized to optimize the power allocation in asymmetric networks (Also, see Property 2 and Appendix B for the high- and low-SNR power allocation in asymmetric networks, respectively). For instance, setting $\phi=20 \mathrm{~dB}, M=2, R=0.5, \epsilon=0.65$ and $\lambda_{1}=\tilde{\lambda}_{1}=1$, Figs. 10a and $10 \mathrm{~b}$ demonstrate the outage probability and the optimal power terms in an asymmetric setup for different fading parameters of the relays. As seen, the system performance is sensitive to the fading parameters when the fading is not severe, i.e., $\lambda_{m}, \tilde{\lambda}_{m}$ 's are small, while its sensitivity decreases with $\lambda_{2}, \tilde{\lambda}_{2}$ (Fig. 10a). Also, as the second relay-destination link experiences severe fading conditions, i.e., $\tilde{\lambda}_{2}$ increases, the source power decreases and more power is allocated to the second relay (Fig. 10b). 


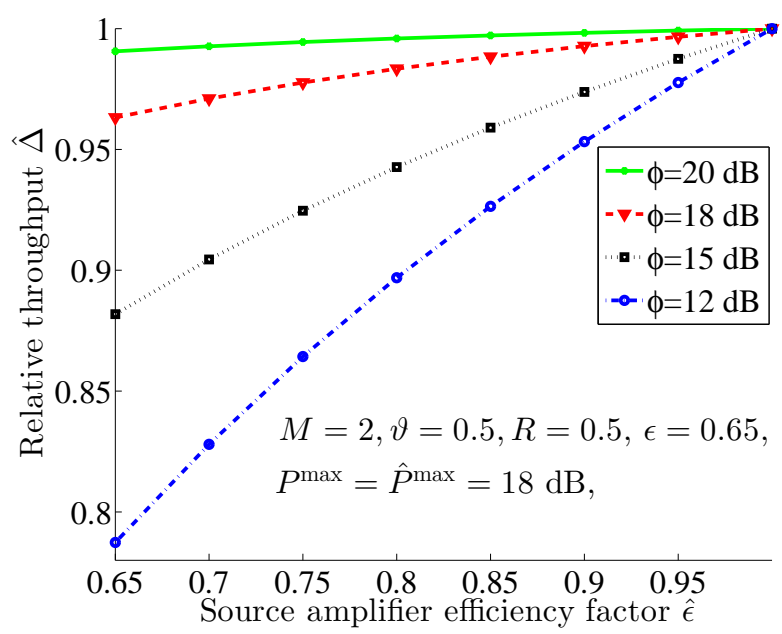

Figure 9. Relative throughput $\hat{\Delta}=\frac{\hat{\eta}}{\eta}$ vs the efficiency factor of the source PA. Rayleigh fading channel, $M=2, R=0.5, \hat{P}^{\max }=P^{\max }=18 \mathrm{~dB}$, $\vartheta=0.5, \epsilon=0.65$.
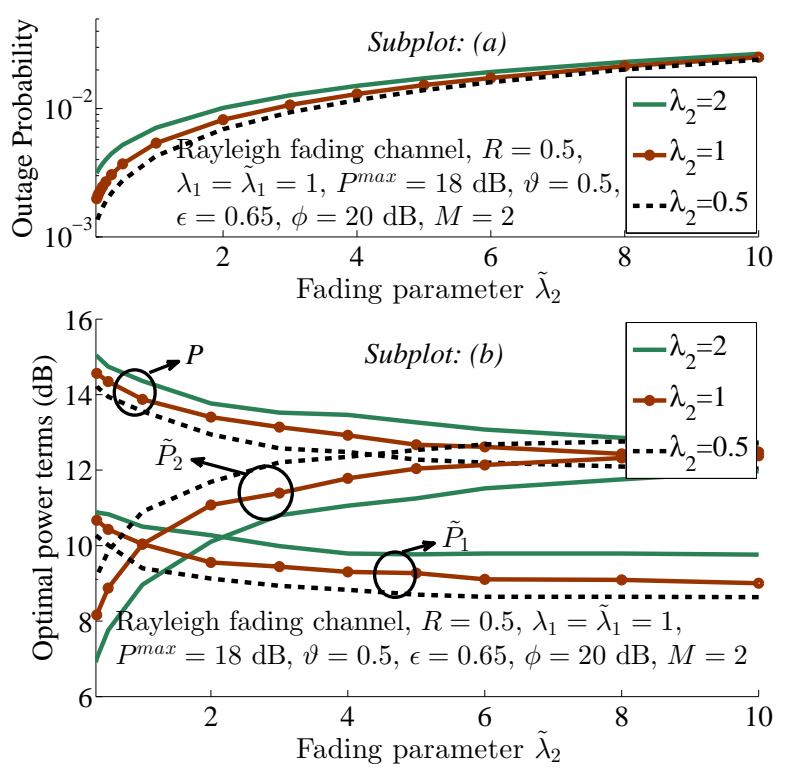

Figure 10. (a) The outage probability and (b) the optimal power terms for different fading parameters $\lambda_{2}, \tilde{\lambda}_{2}$ in an asymmetric setup. Rayleigh fading channel, $M=2, R=0.5, M=2, \lambda=\tilde{\lambda}=1, \hat{P}^{\max }=18 \mathrm{~dB}, \phi=20$ $\mathrm{dB}, \vartheta=0.5, \epsilon=0.65$.

\section{CONCLUSION}

This paper studied the data transmission efficiency of the cooperative and non-cooperative multi-relay networks in the cases where the power amplifiers imperfection is taken into account. We showed numerically and analytically that the inefficiency of the power amplifiers affects the system performance substantially. Particularly, our results emphasize that, with non-ideal power amplifiers at the relays, the diversity and the multiplexing gains of the multi-relay network are zero, and a necessary condition to have positive diversity and multiplexing gains is that the number of relays is scaled with the total SNR at least logarithmically. Moreover, largescale multi-relaying is an effective technique to improve the system performance and compensate the lack of CSI feedback. Finally, the non-cooperative multi-relaying schemes are preferable at low SNRs, because they lead to the same performance as in the cooperative schemes with less signaling overhead/complexity. However, the cooperative schemes outperform the non-cooperative approaches at moderate/high SNRs, and the difference between the two schemes increases with the SNR/number of relays.

Throughout the paper, we studied the effect of the PAs efficiency on the system performance. An interesting topic of research is to analyze the performance of power-adaptive multi-relay networks by taking the PAs nonlinearity/memory into account. Here, the results of [7]-[15], [50], [51] are of great help. Finally, the analysis of the asymmetric large-scale networks is an interesting extension of the paper, for which stochastic geometry [52], [53] is a powerful tool.

\section{APPENDIX A}

\section{PROOF OF PROPERTY 3}

As the second order approximation, either none or at most one of the relays become active at low SNRs, with probabilities $\operatorname{Pr}(\mathcal{M}=\emptyset)=\left(1-e^{-\frac{\lambda\left(e^{R}-1\right)}{P}}\right)^{M} \simeq 1-M e^{-\frac{\lambda\left(e^{R}-1\right)}{P}}$, $\operatorname{Pr}\left(\mathcal{M} \mid C_{\mathcal{M}}=1\right)=\left(1-e^{-\frac{\lambda\left(e^{R}-1\right)}{P}}\right)^{M-1} e^{-\frac{\lambda\left(e^{R}-1\right)}{P}} \simeq$ $e^{-\frac{\lambda\left(e^{R}-1\right)}{P}}$. In this way, using (13), the low-SNR average energy-per-symbol is approximated as

$$
\begin{aligned}
& P+\alpha M \tilde{P}^{1-\vartheta} e^{-\frac{\lambda\left(e^{R}-1\right)}{P}}=\phi \\
& \Rightarrow \tilde{P}=(\phi-P)^{\delta} \alpha^{-\delta} M^{-\delta} e^{\frac{\delta \lambda\left(e^{R}-1\right)}{P}},
\end{aligned}
$$

where $\delta \doteq \frac{1}{1-\vartheta}$.

To maximize the throughput/minimize the outage probability for a given rate $R$, we need to maximize the decoding probability $\operatorname{Pr}($ Decoding $)=1-\operatorname{Pr}($ Outage $)$. Considering the cases with none or at most one successful relay, the decoding probability is given by

$$
\begin{aligned}
& \operatorname{Pr}(\text { Decoding }) \\
& =\sum_{\forall \mathcal{M}, C_{\mathcal{M}}=1} \operatorname{Pr}\left(\mathcal{M} \mid C_{\mathcal{M}}=1\right) \operatorname{Pr}\left(\text { Decoding } \mid \mathcal{M}, C_{\mathcal{M}}=1\right) \\
& =M\left(1-e^{-\frac{\lambda\left(e^{R}-1\right)}{P}}\right)^{M-1} e^{-\frac{\lambda\left(e^{R}-1\right)}{P}} e^{-\frac{\tilde{\lambda}\left(e^{R}-1\right)}{M \tilde{P}}} \\
& =M e^{-\frac{\lambda\left(e^{R}-1\right)}{P}} e^{-\frac{\tilde{\lambda}\left(e^{R}-1\right)}{M \tilde{P}}}
\end{aligned}
$$

where the last equality holds for low SNRs. In this way, from (A.1), the optimization problem (5) is rephrased as

$$
\begin{aligned}
& \left\{\begin{array}{c}
\max _{P, \tilde{P}} e^{-\frac{\lambda\left(e^{R}-1\right)}{P}} e^{-\frac{\tilde{\lambda}\left(e^{R}-1\right)}{M \tilde{P}}} \\
\text { subject to } P+\alpha M \tilde{P}^{1-\vartheta} e^{-\frac{\lambda\left(e^{R}-1\right)}{P}}=\phi,
\end{array}\right. \\
& \equiv\left\{\begin{array}{c}
\min _{P, \tilde{P}} \frac{\lambda}{P}+\frac{\tilde{\lambda}}{M \tilde{P}} \\
\text { subject to } \quad \tilde{P}=(\phi-P)^{\delta} \alpha^{-\delta} M^{-\delta} e^{\frac{\delta \lambda\left(e^{R}-1\right)}{P}},
\end{array}\right.
\end{aligned}
$$

where, considering the low SNR conditions, we have relaxed the relays maximum power constraint. From (A.3), the optimal power allocation is obtained by $\frac{\partial\left(\frac{\lambda}{P}+\tilde{\lambda}(\phi-P)^{-\delta} \alpha^{\delta} M^{\delta-1} e^{-\frac{\delta \lambda\left(e^{R}-1\right)}{P}}\right)}{\partial P}=0$ which leads to 


$$
\begin{aligned}
& \frac{-\lambda}{P^{2}}+\tilde{\lambda} \alpha^{\delta} M^{\delta-1} \times \\
& \left(\delta(\phi-P)^{-\delta-1}+(\phi-P)^{-\delta} \frac{\lambda \delta\left(e^{R}-1\right)}{P^{2}}\right) e^{-\frac{\delta \lambda\left(e^{R}-1\right)}{P}} \\
& \stackrel{(k)}{\simeq} \frac{-\lambda}{P^{2}}+\tilde{\lambda} \alpha^{\delta} \delta M^{\delta-1}(\phi-P)^{-\delta-1} e^{-\frac{\delta \lambda\left(e^{R}-1\right)}{P}} \\
& \stackrel{(l)}{\simeq} \frac{M^{1-\delta} \lambda \phi^{\delta+1} \alpha^{-\delta}}{\tilde{\lambda} \delta P^{2}}-e^{-\frac{\delta \lambda\left(e^{R}-1\right)}{P}}=0 \\
& \left.\stackrel{(m)}{\Rightarrow} P=\frac{\lambda\left(e^{R}-1\right)}{2(1-\vartheta) W\left(\frac{\left(e^{R}-1\right) \sqrt{\lambda \tilde{\lambda}} \alpha^{\frac{1}{2(1-\vartheta)}} M^{\frac{\vartheta}{2(1-\vartheta)}}}{2(1-\vartheta) \sqrt{(1-\vartheta)}} \phi^{\frac{\vartheta-2}{2(1-\vartheta)}}\right.}\right)
\end{aligned}
$$

Here, $(k)$ and $(l)$ are obtained by removing the smallest terms at low SNRs and $(m)$ follows from the definition of Lambert $\mathrm{W}$ function with $e^{-a x}-b x^{2}=0 \Rightarrow x=\frac{2}{a} W\left(\frac{a}{2 \sqrt{b}}\right), \forall a, b \neq 0$. Using (A.1) and (A.4), we find the optimal power term $\tilde{P}$ as in (18).

\section{APPENDIX B}

\section{LOW-SNR POWER Allocation in ASYMmetriC NETWORKS}

Following the same procedure as in the proof of Property 3 , we can derive the optimal power allocation rule of the asymmetric networks at low SNRs. Assuming $\lambda_{i} \neq \lambda_{j}$ and $\tilde{\lambda}_{i} \neq \tilde{\lambda}_{j}, \forall i \neq j,($ A.1) and (A.2) are rephrased as

$$
\bar{P}=P+\alpha \sum_{i=1}^{M} \tilde{P}_{i}^{1-\vartheta} e^{-\frac{\lambda_{i}\left(e^{R}-1\right)}{P}}
$$

and

$$
\operatorname{Pr}(\text { Decoding })=\sum_{i=1}^{M} e^{-\left(\frac{\lambda_{i}}{P}+\frac{\tilde{\lambda}_{i}}{M \tilde{P}_{i}}\right)\left(e^{R}-1\right)},
$$

respectively. Thus, to optimize the throughput/outage probability, we use Lagrangian criterion

$$
\mathcal{Z}=P+\alpha \sum_{i=1}^{M} \tilde{P}_{i}^{1-\vartheta} e^{-\frac{\lambda_{i}\left(e^{R}-1\right)}{P}}+\varpi \sum_{i=1}^{M} e^{-\left(\frac{\lambda_{i}}{P}+\frac{\tilde{\lambda}_{i}}{M \tilde{P}_{i}}\right)\left(e^{R}-1\right)}
$$

where $\varpi$ is the Lagrange multiplier coefficient determined based on $\bar{P}=\phi$. Setting the derivative of the Lagrangian function with respect to $\tilde{P}_{i}$ 's equal to zero, we have

$$
\begin{aligned}
& \frac{\partial \mathcal{Z}}{\partial \tilde{P}_{i}}=0 \\
& \Rightarrow \alpha(\vartheta-1) \tilde{P}_{i}^{2-\vartheta}-\varpi\left(e^{R}-1\right) \frac{\tilde{\lambda}_{i}}{M} e^{-\frac{\tilde{\lambda}_{i}\left(e^{R}-1\right)}{M \tilde{P}_{i}}}=0 \\
& \Rightarrow \tilde{P}_{i}= \\
& \left\lceil\frac{\tilde{\lambda}_{i}\left(e^{R}-1\right)}{M(\vartheta-2) W\left(\frac{1}{\vartheta-2}\left(\frac{\varpi}{\alpha(\vartheta-1)}\left(\frac{\left(e^{R}-1\right) \tilde{\lambda}_{i}}{M}\right)^{\vartheta-1}\right)^{\frac{1}{\vartheta-2}}\right)}\right]^{+},
\end{aligned}
$$

where $\lceil x\rceil^{+} \doteq \max (0, x)$. Also, setting the derivative of (B.3) with respect to $P$ equal to zero leads to

$$
\begin{aligned}
& \frac{\partial \mathcal{Z}}{\partial P}=0 \Rightarrow \mathcal{U}=0, \\
& \mathcal{U} \doteq P^{2}+\alpha \sum_{i=1}^{M} \lambda_{i}\left(e^{R}-1\right) \tilde{P}_{i}^{1-\vartheta} e^{-\frac{\lambda_{i}\left(e^{R}-1\right)}{P}} \\
& +\varpi \sum_{i=1}^{M} \lambda_{i}\left(e^{R}-1\right) e^{-\left(\frac{\lambda_{i}}{P}+\frac{\tilde{\lambda}_{i}}{M \tilde{P}_{i}}\right)\left(e^{R}-1\right)}=0 .
\end{aligned}
$$

Thus, using (B.4), the low-SNR power allocation rule of the asymmetric channels is given by

$$
\left\{\begin{array}{l}
\bar{P}=\phi \\
\mathcal{U}=0
\end{array}\right.
$$

which, because it only has two unknown variables $P$ and $\varpi$, can be efficiently solved by numerical methods.

\section{APPENDIX C}

\section{PROOF OF PROPERTY 4}

The proof follows from the following (in)equalities ${ }^{5}$

$$
\begin{aligned}
r & =\lim _{\phi \rightarrow \infty} \frac{\eta}{\log \phi} \\
& =\lim _{\phi \rightarrow \infty} \frac{R \operatorname{Pr}\left(\frac{1}{M} \sum_{m=1}^{M} \log \left(1+M P^{\max } \tilde{g}_{m}\right) \geq R\right)}{2 \log \phi} \\
& \stackrel{(n)}{\leq} \lim _{\phi \rightarrow \infty} \frac{R e^{-R} E\left\{e^{\left.\log \prod_{m=1}^{M}\left(1+M P^{\max } \tilde{g}_{m}\right)^{\frac{1}{M}}\right\}}\right.}{2 \log \phi} \\
& \stackrel{(o)}{\leq} \lim _{\phi \rightarrow \infty} \frac{e^{-1} E\left\{\prod_{m=1}^{M} \sqrt[M]{1+M P^{\max } \tilde{g}_{m}}\right\}}{2 \log \phi} \\
& \stackrel{(p)}{\leq} \lim _{\phi \rightarrow \infty} \frac{1+M \frac{P^{\max }}{\tilde{\lambda}}}{2 e \log \phi} .
\end{aligned}
$$

Here, $(n)$ comes from the exponential Chebyshev's inequality $\operatorname{Pr}(X \geq x) \leq e^{-t x} E\left\{e^{t X}\right\}, \forall t>0$. Then, $(o)$ is obtained by $R e^{-R} \leq e^{-1}, \forall R \geq 0$, and manipulations. Finally, $(p)$ is given by the Jensen's inequality, the concavity of the $\sqrt[M]{1+x}$ in $x$ and $E\left\{\tilde{g}_{m}\right\}=\frac{1}{\lambda}, \forall m$. According to (C.1), the multiplexing gain is zero, if the number of relays is not scaled by $\phi$ at least logarithmically.

\section{APPENDIX D}

\section{PROOF OF PROPERTY 5}

As mentioned before, the only difference between the two considered cases is in their conditional outage probabilities $\operatorname{Pr}($ Outage $\mid \mathcal{M})$, while $\operatorname{Pr}(\mathcal{M})$, the throughput, the outage probability and the sum consumed energy-per-symbol expressions do not change. Then, as $\log (1+x)=x$ for small $x$ 's, (8) and (31) can be rewritten as

$$
\begin{aligned}
& \operatorname{Pr}(\text { Outage } \mid \mathcal{M})^{\text {non-cooperative }}=\operatorname{Pr}(\text { Outage } \mid \mathcal{M})^{\text {cooperative }} \\
& =\operatorname{Pr}\left(M \sum_{\forall m \in \mathcal{M}} \tilde{g}_{m} \tilde{P}_{m}<R\right),
\end{aligned}
$$

,$\forall i$, at low SNRs. That is, the same throughput and outage probability is achieved by the considered schemes at low SNRs.

${ }^{5}$ The proof is presented for symmetric networks, while the same point is valid for asymmetric setups as well. 


\section{REFERENCES}

[1] N. Bhushan, J. Li, D. Malladi, R. Gilmore, D. Brenner, A. Damnjanovic, R. Sukhavasi, C. Patel, and S. Geirhofer, "Network densification: the dominant theme for wireless evolution into 5G," IEEE Commun. Mag., vol. 52, no. 2, pp. 82-89, Feb. 2014.

[2] O. Oyman, N. Laneman, and S. Sandhu, "Multihop relaying for broadband wireless mesh networks: From theory to practice," IEEE Commun. Mag., vol. 45, no. 11, pp. 116-122, Nov. 2007.

[3] R. Pabst, B. H. Walke, D. C. Schultz, P. Herhold, H. Yanikomeroglu, S. Mukherjee, H. Viswanathan, M. Lott, W. Zirwas, M. Dohler, H. Aghvami, D. Falconer, and G. Fettweis, "Relay-based deployment concepts for wireless and mobile broadband radio," IEEE Commun. Mag., vol. 42, no. 9, pp. 80-89, Sept. 2004.

[4] Y. Jing and H. Jafarkhani, "Single and multiple relay selection schemes and their achievable diversity orders," IEEE Trans. Wireless Commun., vol. 8, no. 3, pp. 1414-1423, Mar. 2009.

[5] K. Vardhe, D. Reynolds, and B. D. Woerner, "Joint power allocation and relay selection for multiuser cooperative communication," IEEE Trans. Wireless Commun., vol. 9, no. 4, pp. 1255-1260, Apr. 2010

[6] V. Genc, S. Murphy, Y. Yu, and J. Murphy, "IEEE 802.16J relaybased wireless access networks: an overview," IEEE Wireless Commun., vol. 15 , no. 5, pp. 56-63, Oct. 2008.

[7] V. del Razo, T. Riihonen, F. Gregorio, S. Werner, and R. Wichman, "Nonlinear amplifier distortion in cooperative amplify-and-forward OFDM systems," in WCNC, Apr. 2009, pp. 1-5.

[8] H. Tan and P. Ho, "Multiply-and-forward - a robust transmission scheme for two-way cooperative communication in the presence of nonlinear power amplifier distortion," in WCNC, Apr. 2013, pp. 4026-4031.

[9] C. Alexandre and R. Fernandes, "Outage performance of cooperative amplify-and-forward OFDM systems with nonlinear power amplifiers," in SPAWC, June 2012, pp. 459-463.

[10] T. Riihonen, S. Werner, F. Gregorio, R. Wichman, and J. Hamalainen, "BEP analysis of OFDM relay links with nonlinear power amplifiers," in $W C N C$, Apr. 2010, pp. 1-6.

[11] I. Ahmad, A. Iyanda Sulyman, A. Alsanie, A. Alasmari, and S. ALshebeili, "Spectral re-growth due to high power amplifier nonlinearities in MIMO-OFDM relaying channels," in IB2Com, Nov. 2011, pp. 240-245.

[12] C. Zhang, Y. Zhang, and Z. Gao, "Performances of amplify-and-forward based wireless relay networks with traveling-wave tube amplifiers," in WCSP, Oct. 2013, pp. 1-5.

[13] J. Qi, S. Aissa, and M.-S. Alouini, "Performance analysis of AF cooperative systems with HPA nonlinearity in semi-blind relays," in GLOBECOM, Dec. 2012, pp. 4182-4186.

[14] C. Zhang, Q. Du, Y. Wang, and G. Wei, "Optimal relay power allocation for amplify-and-forward OFDM relay networks with deliberate clipping," in WCNC, Apr. 2012, pp. 381-386.

[15] M. Haenggi, "The impact of power amplifier characteristics on routing in random wireless networks," in GLOBECOM, vol. 1, Dec. 2003, pp. 513-517 Vol.1.

[16] Ericsson, "Sustainable energy use in mobile communications," www.ericsson.com/technology/whitepapers/sustainable_energy.pdf2007.

[17] P. Gildert, "Power systems efficiency in wireless communication," in The Applied Power Electron Conf., 2006, pp. 1-5.

[18] Https://www.ict-earth.eu/, "EARTH-Energy Aware Radio and neTwork tecHnologies," FP7 project, 2010.

[19] G. Fischer, "Next-Generation Base Station Radio Frequency Architecture," Bell Labs TECH J, vol. 12, pp. 3-18, 2007.

[20] R. Madan, N. B. Mehta, A. F. Molisch, and J. Zhang, "Energy-efficient cooperative relaying over fading channels with simple relay selection," IEEE Trans. Wireless Commun., vol. 7, no. 8, pp. 3013-3025, Aug. 2008.

[21] T. P. Low, M. O. Pun, Y. P. Hong, and C. C. J. Kuo, "Optimized opportunistic multicast scheduling (OMS) over wireless cellular networks," IEEE Trans. Wireless Commun., vol. 9, no. 2, pp. 791-801, Feb. 2010.

[22] S. Mikami, T. Takeuchi, H. Kawaguchi, C. Ohta, and M. Yoshimoto, "An efficiency degradation model of power amplifier and the impact against transmission power control for wireless sensor networks," in RWS, Jan. 2007, pp. 447-450.

[23] E. Bjornemo, "Energy constrained wireless sensor networks: communication principles and sensing aspects," Ph.D. dissertation, Uppsala University, Uppsala, Sweden, 2009.

[24] G. Tsouri and D. Wulich, "Impact of linear power amplifier efficiency on capacity of OFDM systems with clipping," in CEEEI, Dec. 2008, pp. 134-136.

[25] D. Wulich, "Definition of efficient PAPR in OFDM," IEEE Commun. Lett., vol. 9, no. 9, pp. 832-834, Sept. 2005.
[26] H. M. Nemati, C. Fager, and H. Zirath, "High efficiency LDMOS current mode Class-D power amplifier at $1 \mathrm{GHz}$," in European Microwave, Sept. 2006, pp. 176-179.

[27] D. Persson, T. Eriksson, and E. G. Larsson, "Amplifier-aware multipleinput multiple-output power allocation," IEEE Commun. Lett., vol. 17, no. 6, pp. 1112-1115, June 2013.

[28] — "Amplifier-aware multiple-input single-output capacity," IEEE Trans. Commun., vol. 62, no. 3, pp. 913-919, Mar. 2014.

[29] Y. Liu and A. P. Petropulu, "QoS guarantees in AF relay networks with multiple source-destination pairs in the presence of imperfect CSI," IEEE Trans. Wireless Commun., vol. 12, no. 9, pp. 4225-4235, Sept. 2013.

[30] J. Qi and S. Aissa, "Analysis and compensation of power amplifier nonlinearity in MIMO transmit diversity systems," IEEE Trans. Veh. Technol., vol. 59, no. 6, pp. 2921-2931, July 2010.

[31] B. Makki and T. Eriksson, "On the performance of MIMO-ARQ systems with channel state information at the receiver," IEEE Trans. Commun., vol. 62, no. 5, pp. 1588-1603, May 2014.

[32] T. M. Cover and J. A. Thomas, Elements of Information Theory. New York: Wiley Interscience, 1992.

[33] G. Caire and D. Tuninetti, "The throughput of hybrid-ARQ protocols for the Gaussian collision channel," IEEE Trans. Inf. Theory, vol. 47, no. 5, pp. 1971-1988, July 2001.

[34] P. Hesami and J. N. Laneman, "Low-complexity incremental use of multiple transmitters in wireless communication systems," in Allerton, Sept. 2011, pp. 1613-1618.

[35] S. Sesia, G. Caire, and G. Vivier, "Incremental redundancy hybrid ARQ schemes based on low-density parity-check codes," IEEE Trans. Commun., vol. 52, no. 8, pp. 1311-1321, Aug. 2004.

[36] N. Varnica, E. Soljanin, and P. Whiting, "LDPC code ensembles for incremental redundancy hybrid ARQ," in ISIT, Sept. 2005, pp. 995999.

[37] B. Makki, A. Graell i Amat, and T. Eriksson, "Green communication via power-optimized HARQ protocols," IEEE Trans. Veh. Technol., vol. 63, no. 1, pp. 161-177, Jan. 2014.

[38] T. V. K. Chaitanya and E. G. Larsson, "Outage-optimal power allocation for hybrid ARQ with incremental redundancy," IEEE Trans. Wireless Commun., vol. 10, no. 7, pp. 2069-2074, July 2011.

[39] S. Y. Lien, K. C. Chen, Y. C. Liang, and Y. Lin, "Cognitive radio resource management for future cellular networks," IEEE Wireless Commun., vol. 21, no. 1, pp. 70-79, Feb. 2014.

[40] P. Li, S. Guo, W. Zhuang, and B. Ye, "On efficient resource allocation for cognitive and cooperative communications," IEEE J. Sel. Areas Commun., vol. 32, no. 2, pp. 264-273, Feb. 2014.

[41] F. Yilmaz and M.-S. Alouini, "Product of shifted exponential variates and outage capacity of multicarrier systems," in European Wireless, May 2009, pp. 282-286.

[42] A. Chelli, A. Hadjtaieb, and M.-S. Alouini, "Performance analysis of hybrid ARQ with incremental redundancy over amplify-and-forward dualhop relay channels," Submitted to IEEE Trans. on Wireless Commun., 2014, [Online]: http://arxiv.org/abs/1404.2131.

[43] H. El-Gamal, G. Caire, and M.-O. Damen, "The MIMO ARQ channel: Diversity-multiplexing-delay tradeoff," IEEE Trans. Inf. Theory, vol. 52, no. 8, pp. 3601-3621, Aug. 2006.

[44] R. M. Corless, G. H. Gonnet, D. E. G. Hare, D. J. Jeffrey, and D. E. Knuth, "On the Lambert W function," Advances in Computational Mathematics, vol. 5, no. 1, pp. 329-359, 1996.

[45] T. T. Kim and M. Skoglund, "On the expected rate of slowly fading channels with quantized side information," IEEE Trans. Commun., vol. 55, no. 4, pp. 820-829, Apr. 2007.

[46] C. Shen, T. Liu, and M. P. Fitz, "On the average rate performance of hybrid-ARQ in quasi-static fading channels," IEEE Trans. Commun., vol. 57, no. 11, pp. 3339-3352, Nov. 2009.

[47] B. Makki and T. Eriksson, "On hybrid ARQ and quantized CSI feedback schemes in quasi-static fading channels," IEEE Trans. Commun., vol. 60, no. 4, pp. 986-997, Apr. 2012.

[48] _ - "On the ergodic achievable rates of spectrum sharing networks with finite backlogged primary users and an interference indicator signal," IEEE Trans. Wireless Commun., vol. 11, no. 9, pp. 3079-3089, Sept. 2012.

[49] _ - "Feedback subsampling in temporally-correlated slowly-fading channels using quantized CSI," IEEE Trans. Commun., vol. 61, no. 6, pp. 2282-2294, June 2013.

[50] J. Joung, C. K. Ho, K. Adachi, and S. Sun, "A survey on poweramplifier-centric techniques for spectrum and energy efficient wireless communications," IEEE Commun. Surveys Tuts., vol. 17, no. 1, pp. 315333, First quarter 2015. 
[51] J. Joung, C. K. Ho, and S. Sun, "Spectral efficiency and energy efficiency of OFDM systems: Impact of power amplifiers and countermeasures," IEEE J. Sel. Areas Commun., vol. 32, no. 2, pp. 208-220, Feb. 2014.

[52] J. G. Andrews, F. Baccelli, and R. K. Ganti, "A tractable approach to coverage and rate in cellular networks," IEEE Trans. Commun., vol. 59, no. 11 , pp. 3122-3134, Nov. 2011.

[53] S. Lee and K. Huang, "Coverage and economy of cellular networks with many base stations," IEEE Commun. Lett., vol. 16, no. 7, pp. 1038-1040, July 2012.

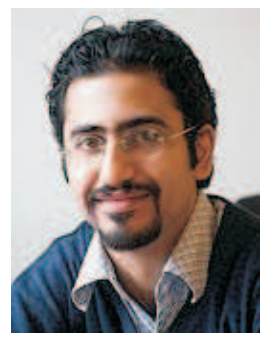

Behrooz Makki was born in Tehran, Iran. He received the B.Sc. degree in Electrical Engineering from Sharif University of Technology, Tehran, Iran, and the M.Sc. degree in Bioelectric Engineering from Amirkabir University of Technology, Tehran, Iran, respectively. Behrooz received his $\mathrm{PhD}$ degree in Communication Engineering from Chalmers University of Technology, Gothenburg, Sweden. Since 2013, he is working as a Postdoc at Chalmers University. Behrooz is the recipient of VR Research Link grant, Sweden, 2014, and the Ericsson's Research grant, Sweden, 2013 and 2014. His current research interests include partial channel state information (CSI) feedback, hybrid automatic repeat request, Green communication, correlated fading channels, spectrum sharing, relay, satellite and CoMP networks.

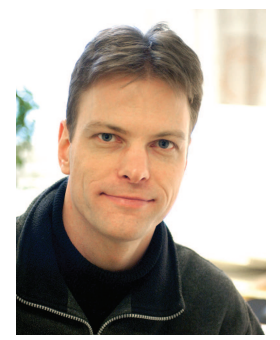

TOMMY SVENSSON [S'98, M'03, SM'10] is Associate Professor in Communication Systems at Chalmers University of Technology, where he is leading the research on air interface and wireless backhaul networking technologies for future wireless systems. He received a Ph.D. in Information theory from Chalmers in 2003, and he has worked at Ericsson $\mathrm{AB}$ with core networks, radio access networks, and microwave transmission products. He was involved in the European collaborative WINNER research projects, and ARTIST4G, which contributed to the 3GPP LTE standards, and he is currently active in the EU FP7 METIS project and the emerging EU H2020 mmMAGIC project targeting solutions for 5G. His main research interests are in design and analysis of physical layer algorithms, multiple access, resource allocation, cooperative systems, moving relays/cells/networks, as well as satellite systems. He has co-authored two books and more than 110 journal and conference papers. He is chairman of the IEEE Sweden Vehicular Technology/Communications/Information Theory chapter, and coordinator of the Communication Engineering Master's Program at Chalmers.

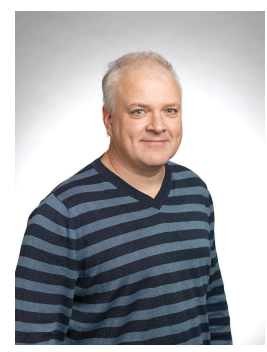

Thomas Eriksson received the Ph.D. degree in Information Theory in 1996, from Chalmers University of Technology, Gothenburg, Sweden. From 1990 to 1996, he was at Chalmers. In 1997 and 1998, he was at AT\&T Labs - Research in Murray Hill, NJ, USA, and in 1998 and 1999 he was at Ericsson Radio Systems AB, Kista, Sweden. Since 1999, he has been at Chalmers University, where he is a professor in communication systems. Further, he was a guest professor at Yonsei University, S. Korea, in 20032004. He is currently vice head of the department of Signals\&Systems at Chalmers, with responsibility for undergraduate and master education. His research interests include communication, data compression, and modeling and compensation of non-ideal hardware components (e.g. amplifiers, oscillators, modulators in communication transmitters and receivers, including massive MIMO).

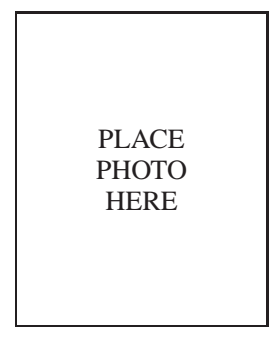

Masoumeh Nasiri-Kenari received her B.S and M.S. degrees in electrical engineering from Isfahan University of Technology, Isfahan, Iran, in 1986 and 1987, respectively, and her Ph.D. degree in electrical engineering from University of Utah, Salt Lake City, in 1993. From 1987 to 1988 , she was a Technical Instructor and Research Assistant at Isfahan University of Technology. Since 1994, she has been with the Department of Electrical Engineering, Sharif University of Technology, Tehran, Iran, where she is now a Professor.

Dr. Nasiri-Kenari founded Wireless Research Laboratory (WRL) of the Electrical Engineering Department in 2001 to coordinate the research activities in the field of wireless communication. Current main activities of the WRL are Cooperative and Cognitive Communications, Network Coding, Molecular Communications, and Green Communications. From 1999-2001, She was a Co-Director of Advanced Communication Research Laboratory, Iran Telecommunication Research Center, Tehran, Iran. She is a recipient of Distinguished Researcher Award and Distinguished Lecturer Award of EE department at Sharif University of Technology in years 2005 and 2007, respectively. She was a Visiting Professor at USC during October 2013-April 2014, where she spent her Sabbatical leave. 